Proceedings of the Edinburgh Mathematical Society (2006) 49, 709-734 (C)

DOI:10.1017/S0013091504001592 Printed in the United Kingdom

\title{
ON ELLIPTIC PROBLEMS IN DOMAINS WITH UNBOUNDED BOUNDARY
}

\author{
JUAN MOLINA ${ }^{1}$ AND RICCARDO MOLLE ${ }^{2}$ \\ ${ }^{1}$ Departamento de Ingenieria Matematica, Facultad de Ciencias Fisicas y Matematicas, \\ Universidad de Conception, Casilla 160-C, Concepcion, Chile \\ (jmolina@ing-mat.udec.cl) \\ ${ }^{2}$ Dipartimento di Matematica, Università di Roma 'Tor Vergata', \\ Via della Ricerca Scientifica n. 1, 00133 Roma, \\ Italy (molle@mat.uniroma2.it)
}

(Received 22 December 2004)

\begin{abstract}
The paper deals with problems of the type $-\Delta u+a(x) u=|u|^{p-2} u, u>0$, with zero Dirichlet boundary condition on unbounded domains in $\mathbb{R}^{N}, N \geqslant 2$, with $a(x) \geqslant c>0, p>2$ and $p<2 N /(N-2)$ if $N \geqslant 3$. The lack of compactness in the problem, related to the unboundedness of the domain, is analysed. Moreover, if the potential $a(x)$ has $k$ suitable 'bumps' and the domain has $h$ suitable 'holes', it is proved that the problem has at least $2(h+k)$ positive solutions ( $h$ or $k$ can be zero). The multiplicity results are obtained under a geometric assumption on $\Omega$ at infinity which ensures the validity of a local Palais-Smale condition.
\end{abstract}

Keywords: unbounded boundary domains; Palais-Smale condition; multiplicity of solutions

2000 Mathematics subject classification: Primary 35J60; 35J20; 35J25

\section{Introduction}

This paper is concerned with problems of the form

$$
\left.\begin{array}{rlrl}
-\Delta u+(1+a(x)) u & =u^{p-1} & & \text { in } \mathcal{D}, \\
u & >0 & & \text { in } \mathcal{D}, \\
u & =0 & & \text { on } \partial \mathcal{D},
\end{array}\right\}
$$$$
P(a, \mathcal{D})
$$

where $\mathcal{D}$ is an unbounded domain in $\mathbb{R}^{N}, p>2, p<2 N /(N-2)$ if $N \geqslant 3$ and $a(x)$ is a non-negative function in $L^{N / 2}(\mathcal{D})$.

Classical arguments show that, when $\mathcal{D}$ is bounded, problem $P(a, \mathcal{D})$ has a solution, whose existence does not depend either on the geometry or on the topology of $\mathcal{D}$. The geometrical-topological properties of $\mathcal{D}$ and the shape of $a$ affect only the multiplicity of solutions; indeed, the number of solutions increases when the structure of $\mathcal{D}$ becomes more complex and when $a$ has more peaks (see, for example, $[\mathbf{4}, \mathbf{5}, \mathbf{8}, \mathbf{1 5}, \mathbf{2 5}]$ ). 
The solutions of problem $P(a, \mathcal{D})$ correspond to the positive functions that are critical points of the energy functional

$$
E_{a}(u)=\int_{\mathcal{D}}\left[|\nabla u|^{2}+(1+a(x)) u^{2}\right] \mathrm{d} x
$$

constrained on the manifold

$$
M(\mathcal{D})=\left\{u \in H_{0}^{1}(\mathcal{D}):|u|_{L^{p}}=1\right\} .
$$

The unboundedness of the domain causes a lack of compactness for $E_{a}$ constrained on $M(\mathcal{D})$, as a consequence of the non-compact embedding $H_{0}^{1}(\mathcal{D}) \hookrightarrow L^{p}(\mathcal{D})$; hence, the topological methods of the calculus of variations do not work directly. This is not only a technical problem. Indeed, there is a large class of domains $\mathcal{D}$ (that includes half-spaces, for instance) in which $P(0, \mathcal{D})$ has no solution (see [13]). On the other hand, it is well known (see $[\mathbf{6}, \mathbf{1 4}, \mathbf{1 6}, \mathbf{2 2}])$ that $P\left(0, \mathbb{R}^{N}\right)$ has a solution and that this solution, which is unique modulo translation, corresponds to the unique radially symmetric function $v_{p}$, $v_{p}>0$, that realizes

$$
m=\min _{u \in M\left(\mathbb{R}^{N}\right)} E_{0}(u) .
$$

When $\mathcal{D}$ is an exterior domain (that is $\mathbb{R}^{N} \backslash \mathcal{D}$ is bounded), $E_{a}$ satisfies the wellknown Palais-Smale compactness condition on $M(\mathcal{D})$ in the energy range $\left(m, 2^{1-2 / p} m\right)$ $($ see $[\mathbf{2}, \mathbf{3}])$ and so it is possible to relate the number of solutions of $P(a, \mathcal{D})$ to the shape of $\mathcal{D}$ and $a$ (see $[\mathbf{2}, \mathbf{3}, \mathbf{7}, \mathbf{9}, \mathbf{1 0}, \mathbf{1 9}-\mathbf{2 1}]$ and references therein).

If not only $\mathcal{D}$ but also $\partial \mathcal{D}$ is unbounded, different situations can occur, from the point of view of the Palais-Smale condition (we refer the reader to [24] for a detailed discussion on the Palais-Smale condition on unbounded domains). For example, in $[\mathbf{1 2}]$ it is proved that, in strip-like domains $\mathcal{D}$, problem $P(0, \mathcal{D})$ has a solution $u_{\mathcal{D}}$; consequently, for these domains the compactness condition cannot hold at level $E_{0}\left(u_{\mathcal{D}} /\left|u_{\mathcal{D}}\right|_{L^{p}}\right)$, because $u_{\mathcal{D}}$ generates a non-compact family of solutions, by translation. Also, for the domains considered in [11], every solution causes a lack of compactness at the corresponding energy level. More precisely, in [11], domains with periodicity in some directions are studied and, after an analysis of the compactness failure, the multiplicity of solutions is proved. In [17], assumptions on the shape of $\mathcal{D}$ at infinity are stated which guarantee a local compactness condition for $E_{a}$ constrained on $M(\mathcal{D})$. Let us remark that, in the examples mentioned above, a non-converging Palais-Smale sequence actually only exists at some energy levels. In $\S 2$ we construct a domain (with unbounded boundary) such that for every energy level $c$ there exists a non-relatively compact Palais-Smale sequence, at level $c$, and we remark that easy examples of domains, thin at infinity, can be given, in which the compactness condition globally holds (see also [18]). In this paper we also want to analyse the effect of the shape of $\mathcal{D}$ and of $a$ on the number of solutions of $P(a, \mathcal{D})$, when $\mathcal{D}$ is a domain with unbounded boundary.

We make the following assumption on the shape of $\mathcal{D}$ at infinity. 
Assumption (C).

$$
\begin{gathered}
\lim _{R \rightarrow+\infty} \inf \{r(x): x \in \mathcal{D},|x|=R\}=+\infty, \\
\lim _{R \rightarrow+\infty} \sup \{h(y): y \in \partial \mathcal{D},|y|=R\}=0,
\end{gathered}
$$

where, for $x \in \mathcal{D}$ and $y \in \partial \mathcal{D}$ we define

$$
\begin{aligned}
& r(x)=\sup \{\rho>0: \exists \bar{x} \in \mathcal{D} \text { such that } x \in B(\bar{x}, \rho) \text { and } B(\bar{x}, \rho) \subset \mathcal{D}\}, \\
& h(y)=\sup \left\{\operatorname{dist}\left(z, T_{\partial \mathcal{D}, y} \cap B(y, 1)\right): z\right. \text { is in the connected component of } \\
& \quad \partial \mathcal{D} \cap B(y, 1) \text { containing } y\},
\end{aligned}
$$

with $B(y, r), r>0$, the ball centred in $y$ with radius $r$, and $T_{\partial \mathcal{D}, y}$ the hyperplane tangent to $\partial \mathcal{D}$ in $y$.

Assumption (C) says that $\mathcal{D}$ enlarges at infinity and that its boundary flattens (or shrinks) at infinity.

The potential $a(x)$ is assumed to decay exponentially. Namely, we suppose that the following condition holds:

$$
\int_{\mathbb{R}^{N}} a(x) \mathrm{e}^{2|x|}\left(1+|x|^{(N-1) \sigma / 2}\right) \mathrm{d} x<+\infty \quad \text { for some } \sigma \in(1,2] .
$$

Now, our goal is to see in which way the presence of 'holes' in the domain and of 'bumps' in the potential affects the number of solutions of $P(a, \mathcal{D})$. To this aim, the effect on the functional $E_{a}$ constrained on $M(\mathcal{D})$ of the holes and the bumps is analysed. Namely, one can see what happens when the holes enlarge, or narrow, and the bumps increase, or vanish. For example, every hole provides a kind of local maximum level in the constrained energy, whose value increases as the size of the hole increases, and the same holds for the bumps in the potential. Moreover, the interaction of two holes (or bumps) produces a saddle-type level in the constrained energy and an analogous effect is given by the interaction of the holes with the bumps and with the 'exterior' boundary of the domain. The saddle-type level related to the interaction of two holes (or bumps) goes down as the holes (bumps) move away from each other. Exploiting the effects described above, it is possible to state that if there are $h$ suitably large holes in the domain and $k$ suitably high bumps in the potential, with appropriate spacing in between them, then our problem has at least $2(h+k)$ solutions (see Theorem 4.1). Note that a key factor in the variational arguments used is a local compactness condition, which is related to Assumption (C) (see Proposition 2.2).

The method used in the proof of Theorem 4.1 can also be employed to cover the case of small holes and small bumps (see $\S 5$ ).

The paper is organized as follows. Section 2 deals with Palais-Smale condition. In $\S 3$ we introduce some tools and preliminary results, which are used in $\S 4$ to prove Theorem 4.1. In $\S 5$ we discuss the asymptotic behaviour of the solutions given by Theorem 4.1 and present other multiplicity results (see Theorem 5.1, for instance). 


\section{The Palais-Smale condition}

In order to find positive functions that are critical points for $E_{a}$ on $M(\mathcal{D})$, we use minimax techniques of the calculus of variations. In general, a basic tool with which to apply these methods is the Palais-Smale compactness condition for $E_{a}$ on $M(\mathcal{D})$. When $\mathcal{D}$ is bounded, it is well known that global compactness holds, as a consequence of the Rellich's compact embedding theorem, and variational methods work (see, for example, [23, §§ II-2, II-6]). Actually, to obtain the Palais-Smale condition it is sufficient to have

$$
H_{0}^{1}(\mathcal{D}) \hookrightarrow L^{p}(\mathcal{D})
$$

compact. Hence, taking into account [1, Theorem 6.16], for instance, if $\mathcal{D}$ becomes thin at infinity, then compactness holds. As an example, the following domain can be considered:

$$
\begin{aligned}
\mathcal{D}_{0}=\left\{x=\left(x_{1}, \ldots, x_{N}\right) \in \mathbb{R}^{N}: \sum_{i=1}^{N-1}\left|x_{i}\right|^{2}\right. & \left.<\frac{1}{1+\left|x_{N}\right|}\right\} \\
& \cup \bigcup_{n=2}^{+\infty}\left\{x \in \mathbb{R}^{N}: n<|x|<n+\frac{1}{n}\right\} .
\end{aligned}
$$

While in $\mathcal{D}_{0}$ every Palais-Smale sequence at every energy level is relatively compact, the following example provides a domain in which, conversely, for every energy level there exist non-relatively compact Palais-Smale sequences.

Example 2.1. Let $\left(q_{i}\right)_{i}=\mathbb{Q}^{+}$and define

$$
S_{i}=\left\{\left(x_{1}, \ldots, x_{N}\right) \in \mathbb{R}^{N}:-\frac{1}{2} q_{i}<x_{1}<\frac{1}{2} q_{i}\right\} .
$$

Then set

$$
\begin{aligned}
& \hat{\mathcal{D}}=\left\{x=\left(x_{1}, \ldots, x_{N}\right) \in \mathbb{R}^{N}:\right.\left.\sum_{j=2}^{N} x_{j}^{2}<1\right\} \\
& \cup S_{1} \cup\left[\bigcup_{i=2}^{\infty}\left[S_{i}+\left(i+\sum_{j=1}^{i-1} q_{j}+\frac{1}{2} q_{i}, 0, \ldots, 0\right)\right]\right] .
\end{aligned}
$$

For every $a \in L^{N / 2}(\hat{\mathcal{D}}), E_{a}(M(\hat{\mathcal{D}}))=(m,+\infty)$ holds and, for every $c \in[m,+\infty)$, there exists a Palais-Smale sequence for $E_{a}$ constrained on $M(\hat{\mathcal{D}})$, at level $c$, that is not relatively compact.

To prove our assertions, let us recall that for every $i \in \mathbb{N}$ there exists a critical point $u_{i}$ for $E_{0}$ on $M\left(S_{i}\right)$, corresponding to the 'minimal' solution of $P\left(0, S_{i}\right)$ (see [12]). Namely, $u_{i}$ satisfies

$$
\Theta\left(q_{i}\right):=\min _{M\left(S_{i}\right)} E_{0}(u)=E_{0}\left(u_{i}\right)
$$


From (2.2) it follows that the map $\Theta: \mathbb{Q} \rightarrow \mathbb{R}$ is a monotone decreasing continuous function and we claim that

$$
\begin{aligned}
\lim _{q_{i} \rightarrow+\infty} E_{0}\left(u_{i}\right) & =m, \\
\lim _{q_{i} \rightarrow 0} E_{0}\left(u_{i}\right) & =+\infty .
\end{aligned}
$$

Taking into account (2.2), to prove (2.3) it is sufficient to construct, for every $i \in \mathbb{N}$, a function $w_{i} \in M\left(S_{i}\right)$ such that

$$
\lim _{q_{i} \rightarrow+\infty} E_{0}\left(w_{i}\right)=m
$$

It is well known that the unique radially symmetric function $v_{p}$ that realizes (1.1) satisfies

$$
\begin{gathered}
\lim _{|x| \rightarrow+\infty}\left|v_{p}(x)\right||x|^{(N-1) / 2} \mathrm{e}^{|x|}=d>0, \\
\lim _{|x| \rightarrow+\infty}\left|\nabla v_{p}(x)\right||x|^{(N-1) / 2} \mathrm{e}^{|x|}=d,
\end{gathered}
$$

for a suitable positive constant $d$ (see [6]). Moreover, for every $i \in \mathbb{N}$ let us introduce the function $c_{i}: \mathbb{R}^{N} \rightarrow[0,1]$ defined by $c_{i}(x)=f\left(|x| / q_{i}\right)$, where $f \in C^{\infty}\left(\mathbb{R}^{+},[0,1]\right)$ is a non-increasing function such that

$$
f \equiv \begin{cases}1 & \text { in }\left[0, \frac{1}{4}\right] \\ 0 & \text { in }\left[\frac{1}{2},+\infty\right)\end{cases}
$$

From (2.6) and (2.7) it follows that

$$
\lim _{q_{i} \rightarrow+\infty} c_{i} v_{p}=v_{p} \quad \text { in } L^{p}\left(\mathbb{R}^{N}\right) \text { and in } H^{1}\left(\mathbb{R}^{N}\right) .
$$

So, (2.5) follows by setting $w_{i}=c_{i} v_{p} /\left|c_{i} v_{p}\right|_{L^{p}}$.

To prove (2.4) observe that, by the Poincaré inequality on a strip, there exists a constant $\bar{c}>0$ such that

$$
|u|_{L^{p}} \leqslant \bar{c}|\nabla u|_{L^{2}}, \quad \forall u \in H_{0}^{1}\left(S_{1}\right)
$$

(see [1, Theorem 6.30], for example). To simplify the notation, let us assume $q_{1}=1$ and set $\hat{u}_{i}(x)=u_{i}\left(q_{i} x\right)$; applying $(2.9)$ to $\hat{u}_{i}$ we get

$$
q_{i}^{-[2 N-p(N-2)] /(2 p)} \leqslant \bar{c}\left|\nabla u_{i}\right|_{L^{2}\left(S_{q_{i}}\right)},
$$

because $\left|u_{i}\right|_{L^{p}}=1 \forall i \in \mathbb{N}$, which implies our claim.

Now, if $c=\Theta\left(q_{i}\right)$ for some $i \in \mathbb{N}$, let us construct the sequence $\left(u_{i, j}\right)_{j}$ in $M(\hat{\mathcal{D}})$ by

$$
u_{i, j}\left(x_{1}, \ldots, x_{N}\right)=u_{i}\left(x_{1}-\left(i+\sum_{r=1}^{i-1} q_{r}+\frac{1}{2} q_{i}\right), x_{2}+j, x_{3}, \ldots, x_{N}\right)
$$


( $u_{i}$ is extended by 0 outside $S_{i}$ ). It is not then difficult to see that $\left(u_{i, j}\right)_{j}$ is a PalaisSmale sequence for $E_{0}$ constrained on $M(\hat{\mathcal{D}})$, at level $c$, and it cannot have a converging subsequence.

If $c \in[m,+\infty) \backslash \Theta(\mathbb{Q})$, let $\left(q_{i_{j}}\right)_{j}$ be a sequence in $\mathbb{Q}$ such that $c=\lim _{j \rightarrow+\infty} \Theta\left(q_{i_{j}}\right)$. Then $\left(u_{i_{j}, j}\right)_{j}$ is a Palais-Smale sequence for $E_{0}$ constrained on $M(\hat{\mathcal{D}})$ at level $c$ that has no converging subsequence.

The equality $(m,+\infty)=E_{0}(M(\hat{\mathcal{D}}))$ is a direct consequence of $(1.1),(2.3),(2.4)$ and of the continuity of $E_{0}$.

Now, to conclude, it is sufficient to observe that, for every fixed non-negative $a \in$ $L^{N / 2}(\hat{\mathcal{D}}), E_{a}(u) \geqslant E_{0}(u) \forall u \in H_{0}^{1}(\hat{\mathcal{D}})$ and

$$
\begin{aligned}
\lim _{j \rightarrow+\infty}\left|E_{a}\left(u_{i, j}\right)-E_{0}\left(u_{i, j}\right)\right|=0, & \forall i \in \mathbb{N}, \\
\lim _{j \rightarrow+\infty}\left|E_{a}\left(u_{i_{j}, j}\right)-E_{0}\left(u_{i_{j}, j}\right)\right|=0, & \\
\lim _{j \rightarrow+\infty} \nabla E_{a}\left(u_{i, j}\right)-\nabla E_{0}\left(u_{i, j}\right)=0, & \forall i \in \mathbb{N}, \\
\lim _{j \rightarrow+\infty} \nabla E_{a}\left(u_{i_{j}, j}\right)-\nabla E_{0}\left(u_{i_{j}, j}\right)=0 & \text { in } H^{-1}(\hat{\mathcal{D}}) .
\end{aligned}
$$

In the domains we consider, we cannot expect a nice situation, as in (2.1), from the point of view of compactness, but, because of Assumption (C), the situation is also not as bad as in Example 2.1. In fact, we have the following local compactness result.

Proposition 2.2. Assume that $\mathcal{D} \subset \mathbb{R}^{N}$ satisfies Assumption $(C)$ and let a be a nonnegative function in $L^{N / 2}(\mathcal{D})$. If $\left(u_{n}\right)_{n}$ is a Palais-Smale sequence for $E_{a}$ constrained on $M(\mathcal{D})$ at a level $c \in\left(m, 2^{1-2 / p} m\right)$, then $\left(u_{n}\right)_{n}$ is relatively compact.

Proof. The proof can be obtained arguing exactly as in the proof of [17, Lemma 3.1].

By using Proposition 2.2, in the proof of Theorem 4.1 we will find $2(h+k)$ distinct critical values for $E_{a}$ on $M(\mathcal{D})$ in the energy range $\left(m, 2^{1-2 / p} m\right)$. The following proposition states that the critical points that correspond to critical values in $\left(m, 2^{1-2 / p} m\right)$ are functions that do not change sign in $\mathcal{D}$; actually, they have constant sign, by the maximum principle, and so solve $P(a, \mathcal{D})$.

Proposition 2.3. Let $\mathcal{D}$ be an open set in $\mathbb{R}^{N}$ and let a be a non-negative function in $L^{N / 2}(\mathcal{D})$. If $u \in H_{0}^{1}(\mathcal{D})$ is such that

$$
|u|_{L^{p}}=1, \quad E_{a}(u)=c, \quad \nabla E_{a \mid M(\mathcal{D})}(u)=0,
$$

then $u^{+} \not \equiv 0$ and $u^{-} \not \equiv 0$ implies $c>2^{1-2 / p} m$.

Proof. The proof is contained in the proof of [9, Theorem 1.1] and can be deduced by the minimality of $m$ (see (1.1)).

Finally, observe that, in our domains, the Palais-Smale condition cannot hold at level $m$ and that $P(a, \mathcal{D})$ cannot be solved by a minimization argument, because of the following result. 
Proposition 2.4. Let $\mathcal{D}$ be an open set in $\mathbb{R}^{N}$ satisfying $\left(\mathrm{C}_{1}\right)$ and $a(x)$ be a nonnegative function in $L^{N / 2}\left(\mathbb{R}^{N}\right)$. Then

$$
\inf _{M(\mathcal{D})} E_{a}=m
$$

and, if $\mathcal{D} \neq \mathbb{R}^{N}$ or $a \neq \equiv$, the infimum in (2.10) is not achieved.

Proof. Observe that, by $\left(\mathrm{C}_{1}\right)$, for every $n \in \mathbb{N}$ there exists $z_{n} \in \mathcal{D}$ such that $B\left(z_{n}, n\right) \subset \mathcal{D}$. Then, arguing as for $(2.5)$, we have

$$
\inf _{u \in M(\mathcal{D})} E_{a}(u)=m
$$

If $u^{*} \in M(\mathcal{D})$ realizes $(2.11)$, then $u^{*}$ also realizes $(1.1)$, because

$$
m \leqslant E_{0}\left(u^{*}\right) \leqslant E_{a}\left(u^{*}\right)=m .
$$

Then, by the uniqueness of the minimizers of (1.1), there exists $y^{*} \in \mathbb{R}^{N}$ such that $u^{*}(x)=v_{p}\left(x-y^{*}\right)$. Since $v_{p}>0$ in $\mathbb{R}^{N}$, we can conclude that $\mathcal{D}=\mathbb{R}^{N}$. Moreover,

$$
\begin{aligned}
m=E_{a}\left(u^{*}\right) & =\int_{\mathbb{R}^{N}}\left[\left|\nabla v_{p}\left(x-y^{*}\right)\right|^{2}+(1+a(x)) v_{p}^{2}\left(x-y^{*}\right)\right] \mathrm{d} x \\
& =\int_{\mathbb{R}^{N}}\left[\left|\nabla v_{p}\left(x-y^{*}\right)\right|^{2}+v_{p}^{2}\left(x-y^{*}\right)\right] \mathrm{d} x+\int_{\mathbb{R}^{N}} a(x) v_{p}^{2}\left(x-y^{*}\right) \mathrm{d} x \\
& =m+\int_{\mathbb{R}^{N}} a(x) v_{p}^{2}\left(x-y^{*}\right) \mathrm{d} x
\end{aligned}
$$

which implies that $a \equiv 0$ because $a$ is non-negative.

\section{Tools, preliminary results and known facts}

For every smooth domain $\mathcal{D} \subset \mathbb{R}^{N}$, we define a cut-off function $c_{\mathcal{D}}$, which is a function in $C^{\infty}\left(\mathbb{R}^{N},[0,1]\right)$ such that $c_{\mathcal{D}}=0$ on $\mathbb{R}^{N} \backslash \mathcal{D}, c_{\mathcal{D}}(x)=1$ if $x \in \mathcal{D}$ and $\operatorname{dist}(x, \partial \mathcal{D}) \geqslant 1$. If $\mathcal{D}=\mathbb{R}^{N}$, set $c_{\mathcal{D}} \equiv 1$.

Then we introduce the map $v_{p, \mathcal{D}}: \mathbb{R}^{N} \rightarrow M(\mathcal{D})$ as

$$
v_{p, \mathcal{D}}[y](x)=\frac{c_{\mathcal{D}}(x) v_{p}(x-y)}{\left|c_{\mathcal{D}} v_{p}(\cdot-y)\right|_{L^{p}}} .
$$

Fixing $\zeta \in \partial B(0,1)$ and $z \in \mathbb{R}^{N}$, we define

$$
\Sigma_{z}=\partial B(z+\zeta, 2)=\left\{y \in \mathbb{R}^{N}:|y-(z+\zeta)|=2\right\}
$$

and, for $\rho>0$, we define

$$
\Psi_{\mathcal{D}, \rho, z}: \Sigma_{z} \times[0,1] \rightarrow M(\mathcal{D})
$$

as

$$
\Psi_{\mathcal{D}, \rho, z}[y, t](x)=\frac{c_{\mathcal{D}}(x)\left[(1-t) v_{p}(x-[z+\rho(y-z)])+t v_{p}(x-(z+\rho \zeta))\right]}{\left|c_{\mathcal{D}}\left[(1-t) v_{p}(\cdot-[z+\rho(y-z)])+t v_{p}(\cdot-(z+\rho \zeta))\right]\right|_{L^{p}}}
$$


Notice that

$$
\Psi_{\mathcal{D}, \rho, z}[y, 0]=v_{p, \mathcal{D}}[z+\rho(y-z)], \quad \Psi_{\mathcal{D}, \rho, z}[y, 1]=v_{p, \mathcal{D}}[z+\rho \zeta], \quad \forall z \in \mathbb{R}^{N}, \forall y \in \Sigma_{z} .
$$

For $z \in \mathbb{R}^{N}$, let $\beta_{z}: L^{p}\left(\mathbb{R}^{N}\right) \backslash\{0\} \rightarrow \mathbb{R}^{N}$ be the function defined by

$$
\beta_{z}(u)=z+\frac{1}{|u|_{L^{p}}^{p}} \int_{\mathbb{R}^{N}} \chi(x-z)|u(x)|^{p} \mathrm{~d} x
$$

where

$$
\chi(x)=\frac{x}{1+|x|}
$$

For $u \in L^{p}\left(\mathbb{R}^{N}\right)$ we set

$$
\tilde{u}(x)=\frac{1}{\omega_{N}} \int_{B(x, 1)}|u(y)| \mathrm{d} y,
$$

where $\omega_{N}$ is the measure of the unit ball in $\mathbb{R}^{N}$, and

$$
\hat{u}(x)=\left[\tilde{u}(x)-\frac{1}{2} \max _{\mathbb{R}^{N}} \tilde{u}(x)\right]^{+} .
$$

We then define the map

$$
\beta: L^{p}\left(\mathbb{R}^{N}\right) \backslash\{0\} \rightarrow \mathbb{R}^{N}
$$

by

$$
\beta(u)=\frac{1}{|\hat{u}|_{L^{p}}^{p}} \int_{\mathbb{R}^{N}}[\hat{u}(x)]^{p} x \mathrm{~d} x .
$$

The 'barycentre-type' functions $\beta_{z}$ and $\beta$ are well defined and continuous in the $L^{p}$-norm. Moreover,

$$
\beta(u(x-\bar{x}))=\beta(u)+\bar{x}, \quad \forall u \in L^{p}\left(\mathbb{R}^{N}\right) \backslash\{0\}, \forall \bar{x} \in \mathbb{R}^{N},
$$

and, by the radial symmetry of $v_{p}$,

$$
\beta\left(v_{p}(\cdot-y)\right)=y, \quad \forall y \in \mathbb{R}^{N} .
$$

Finally, for $x, y \in \mathbb{R}^{N}$, we denote the segment joining $x$ and $y$ by

$$
[x, y]=\{x+t(y-x): t \in[0,1]\} .
$$

Proposition 3.1. Let $a(x)$ be a non-negative function in $L^{N / 2}\left(\mathbb{R}^{N}\right)$, and $\Omega$ and $\omega$ be open domains in $\mathbb{R}^{N}, \bar{\Omega}, \omega \neq \mathbb{R}^{N}$. If $a(x) \not \equiv 0$ or $\omega \neq \emptyset$, then there exists $\mu>m$ such that

$$
\begin{aligned}
& \inf \left\{E_{a(-\bar{x})}(u): \bar{x} \in \mathbb{R}^{N}, u \in M(\Omega \backslash(\bar{\omega}+\bar{x})), \beta(u)=0\right\}>\mu, \\
& \inf \left\{E_{a(\cdot-\bar{x})}(u): \bar{x} \in \mathbb{R}^{N}, u \in M(\Omega \backslash(\bar{\omega}+\bar{x})), \beta(u)=\bar{x}\right\}>\mu
\end{aligned}
$$

(with the notation $\inf \emptyset=+\infty$ ). 
Proof. Since $M(\Omega \backslash(\bar{\omega}+\bar{x})) \subseteq M(\Omega)$ and $E_{a(\cdot-\bar{x})}(u) \geqslant E_{0}(u)$, for all $u \in H^{1}\left(\mathbb{R}^{N}\right)$ and all $\bar{x} \in \mathbb{R}^{N}$, in order to prove (3.6) it is sufficient to verify that

$$
\inf \left\{E_{0}(u): u \in M(\Omega), \beta(u)=0\right\}>m .
$$

Assume, by contradiction, that (3.8) does not hold, i.e. there exists a sequence $\left(u_{n}\right)_{n}$ in $M(\Omega)$ such that $\beta\left(u_{n}\right)=0$ for all $n \in \mathbb{N}$ and $\lim _{n \rightarrow+\infty} E_{0}\left(u_{n}\right)=m$. Since $\left(u_{n}\right)_{n}$ is a minimizing sequence for (1.1), there exists, as proved in [3], a sequence of points $\left(y_{n}\right)_{n}$ in $\mathbb{R}^{N}$ and a sequence of functions $\left(w_{n}\right)_{n}$ in $H^{1}\left(\mathbb{R}^{N}\right)$ such that

$$
u_{n}(x)=v_{p}\left(x-y_{n}\right)+w_{n}(x) \text { with } \lim _{n \rightarrow+\infty}\left\|w_{n}\right\|_{H^{1}}=0 .
$$

The sequence $\left(y_{n}\right)_{n}$ has to be unbounded. Indeed, if $y_{n} \underset{n \rightarrow+\infty}{\longrightarrow} \bar{y}$, up to a subsequence, then from (3.9) it follows that

$$
\lim _{n \rightarrow+\infty} E_{0}\left(u_{n}\right)=\int_{\Omega}\left[\left|\nabla v_{p}(x-\bar{y})\right|^{2}+\left(v_{p}(x-\bar{y})\right)^{2}\right] \mathrm{d} x<m,
$$

because the function $v_{p}$ that realizes (1.1) is strictly positive on $\mathbb{R}^{N}$, while $\bar{\Omega} \neq \mathbb{R}^{N}$; hence, a contradiction arises.

Observe that, from (3.4), (3.5) and the continuity of $\beta$, it follows that

$$
\begin{aligned}
\lim _{n \rightarrow+\infty}\left|\beta\left(u_{n}\right)-y_{n}\right| & =\lim _{n \rightarrow+\infty}\left|\beta\left(v_{p}\left(x-y_{n}\right)+w_{n}(x)\right)-y_{n}\right| \\
& =\lim _{n \rightarrow+\infty}\left|\beta\left(v_{p}(x)+w_{n}\left(x+y_{n}\right)\right)\right|=0,
\end{aligned}
$$

and this contradicts the fact that $\left(y_{n}\right)_{n}$ is unbounded, because $\beta\left(u_{n}\right) \equiv 0$. So (3.8), and thus (3.6), is proved.

Taking into account $(3.4)$ and $M((\Omega-\bar{x}) \backslash \bar{\omega}) \subset M\left(\mathbb{R}^{N} \backslash \bar{\omega}\right)$, in order to prove (3.7) it is sufficient to verify that

$$
\inf \left\{E_{a}(u): u \in M\left(\mathbb{R}^{N} \backslash \bar{\omega}\right), \beta(u)=0\right\}>m .
$$

If $\omega \neq \emptyset$, then (3.12) can be proved by arguing as for the proof of (3.8). Therefore, assume that $\omega=\emptyset$ and suppose, by contradiction, that (3.12) does not hold, i.e. there exists a sequence $\left(v_{n}\right)_{n}$ in $H^{1}\left(\mathbb{R}^{N}\right)$ such that

$$
E_{a}\left(v_{n}\right) \underset{n \rightarrow+\infty}{\longrightarrow} m
$$

and $\beta\left(v_{n}\right)=0$ for all $n \in \mathbb{N}$. Since $m \leqslant E_{0}\left(v_{n}\right) \leqslant E_{a}\left(v_{n}\right)$, because $m=\min _{M\left(\mathbb{R}^{N}\right)} E_{0}$ and $a(x) \geqslant 0$ for all $x \in \mathbb{R}^{N}$, we find that $\left(v_{n}\right)_{n}$ is a minimizing sequence for (1.1). There then exists a sequence of points $\left(y_{n}\right)$ in $\mathbb{R}^{N}$ and a sequence of functions $\left(w_{n}\right)_{n}$ in $H^{1}\left(\mathbb{R}^{N}\right)$ such that (3.9) holds with $v_{n}$ in place of $u_{n}$. The sequence $\left(y_{n}\right)_{n}$ is unbounded, otherwise $y_{n} \underset{n \rightarrow+\infty}{\longrightarrow} \bar{y}$, up to a subsequence, and

$$
\lim _{n \rightarrow+\infty} E_{a}\left(v_{n}\right)=\int_{\mathbb{R}^{N}}\left[\left|\nabla v_{p}(x-\bar{y})\right|^{2}+(1+a(x))\left(v_{p}(x-\bar{y})\right)^{2}\right] \mathrm{d} x>m,
$$


because $v_{p}>0$ in $\mathbb{R}^{N}$ and $a \not \equiv 0$. But $\left(y_{n}\right)_{n}$ cannot be unbounded; indeed, arguing as in (3.11), we find that

$$
\left|\beta\left(v_{n}\right)-y_{n}\right| \underset{n \rightarrow+\infty}{\longrightarrow} 0
$$

and that $\beta\left(v_{n}\right) \equiv 0$ holds. So a contradiction arises and (3.12) is proved.

Proposition 3.2. Let $\Omega$ and $\omega$ be open domains in $\mathbb{R}^{N}$ and let $a \in L^{q}\left(\mathbb{R}^{N}\right)$ for some $q \in[1,+\infty)$; moreover, assume that $\omega$ is bounded and that $\Omega$ verifies $\left(\mathrm{C}_{1}\right)$. If $\left(z_{n}\right)_{n}$ is such that $B\left(z_{n}, n\right) \subset \Omega$, then
(a) $\lim _{n \rightarrow+\infty} \sup _{y \in \partial B\left(z_{n}, n / 2\right)} E_{a\left(\cdot-z_{n}\right)}\left(v_{p, \Omega \backslash\left(\bar{\omega}+z_{n}\right)}[y]\right)=m$,
(b) $\lim _{n \rightarrow+\infty} \sup _{y \in \partial B\left(z_{n}, n / 2\right)}\left|\beta\left(v_{p, \Omega \backslash\left(\bar{\omega}+z_{n}\right)}[y]\right)-y\right|=0$.

Proof. From (2.6) and (2.7) we may infer that

$$
\begin{aligned}
& \lim _{n \rightarrow+\infty} \sup _{y \in \partial B\left(z_{n}, n / 2\right)}\left|v_{p}(\cdot-y)-c_{\Omega \backslash\left(\bar{\omega}+z_{n}\right)} v_{p}(\cdot-y)\right|_{L^{p}}=0, \\
& \lim _{n \rightarrow+\infty} \sup _{y \in \partial B\left(z_{n}, n / 2\right)}\left\|v_{p}(\cdot-y)-c_{\Omega \backslash\left(\bar{\omega}+z_{n}\right)} v_{p}(\cdot-y)\right\|_{H^{1}}=0 ;
\end{aligned}
$$

therefore

$$
\lim _{n \rightarrow+\infty} \sup _{y \in \partial B\left(z_{n}, n / 2\right)}\left\|v_{p}(\cdot-y)-v_{p, \Omega \backslash\left(\bar{\omega}+z_{n}\right)}[y]\right\|_{H^{1}}=0 .
$$

Moreover, we claim that

$$
\int_{\mathbb{R}^{N}} a(x-\bar{z}) v_{p}^{2}(x-\bar{y}) \mathrm{d} x=\int_{\mathbb{R}^{N}} a(x) v_{p}^{2}(x-(\bar{y}-\bar{z})) \mathrm{d} x \rightarrow 0 \quad \text { as } \quad|\bar{y}-\bar{z}| \rightarrow+\infty .
$$

Indeed, assume that $q>1$ and call $q^{\prime}=q /(q-1)$ the conjugate exponent. Let us fix $\eta>0$ and choose $R$ to be large enough to have

$$
|a|_{L^{q}\left(\mathbb{R}^{N} \backslash B(0, R)\right)}<(\eta / 2)\left|v_{p}^{2}\right|_{L^{q^{\prime}}\left(\mathbb{R}^{N}\right)}^{-1}
$$

and let $|\bar{y}-\bar{z}|$ be large enough to have

$$
\left|v_{p}^{2}\right|_{L^{q^{\prime}(B(\bar{z}-\bar{y}, R))}}<(\eta / 2)|a|_{L^{q}\left(\mathbb{R}^{N}\right)}^{-1}
$$

(from (2.6), it follows that $v_{p}^{2} \in L^{r}\left(\mathbb{R}^{N}\right)$ for all $r \in[1,+\infty)$ ). Then

$$
\begin{aligned}
\int_{\mathbb{R}^{N}} a(x) v_{p}^{2}(x-(\bar{y}-\bar{z})) \mathrm{d} x= & \int_{B(0, R)} a(x) v_{p}^{2}(x-(\bar{y}-\bar{z})) \mathrm{d} x \\
& \quad+\int_{\mathbb{R}^{N} \backslash B(0, R)} a(x) v_{p}^{2}(x-(\bar{y}-\bar{z})) \mathrm{d} x \\
\leqslant & |a|_{L^{q}\left(\mathbb{R}^{N}\right)}\left|v_{p}^{2}\right|_{L^{q^{\prime}}(B(\bar{z}-\bar{y}, R))}+|a|_{L^{q}\left(\mathbb{R}^{N} \backslash B(0, R)\right)}\left|v_{p}^{2}\right|_{L^{q^{\prime}}\left(\mathbb{R}^{N}\right)} \\
\leqslant &
\end{aligned}
$$

and (3.16) follows on letting $\eta \rightarrow 0$. 
If $q=1$, then (3.16) follows from the Lebesgue theorem and (2.6).

By using (3.16) and (3.15) we obtain

$$
\lim _{n \rightarrow+\infty} \sup _{y \in \partial B\left(z_{n}, n / 2\right)} \int_{\mathbb{R}^{N}} a\left(x-z_{n}\right)\left(v_{p, \Omega \backslash\left(\bar{\omega}+z_{n}\right)}(x-y)\right)^{2} \mathrm{~d} x=0 .
$$

Assertion (a) follows from (3.15) and (3.17).

By (3.15) we have

$$
\lim _{n \rightarrow+\infty} \sup _{y \in \partial B\left(z_{n}, n / 2\right)}\left|v_{p, \Omega \backslash\left(\bar{\omega}+z_{n}\right)}[y]-v_{p}(\cdot-y)\right|_{L^{p}}=0,
$$

and hence (b) follows from the continuity of $\beta$ and from (3.5).

Remark 3.3. By Proposition 3.2 (b) we find that, for $n$ large enough, there exists $y \in \partial B\left(z_{n}, \frac{1}{2} n\right)$ such that $\beta\left(v_{p, \Omega \backslash\left(\bar{\omega}+z_{n}\right)}[y]\right) \in\left[0, z_{n}\right]$.

The same arguments used to prove Propositions 2.4, 3.1 and 3.2 can be used to state the following results, where the effects on the energy functional of the 'holes' in the domain and of the bumps in the potential are described. In particular, these results show that the holes and the bumps play the same role.

Proposition 3.4. For $x_{1}, x_{2} \in \mathbb{R}^{N}$ and $D_{1}, D_{2}$ bounded non-empty open sets in $\mathbb{R}^{N}$, define $\mathcal{D}=\mathbb{R}^{N} \backslash \bigcup_{i=1}^{2}\left(\bar{D}_{i}+x_{i}\right)$. There then exists $\mu_{D_{1}, D_{2}}>m$ such that

$$
\inf _{x_{1}, x_{2} \in \mathbb{R}^{N}} \inf \left\{E_{0}(u): u \in M(\mathcal{D}), \beta(u) \in\left\{x_{1}, x_{2}\right\}\right\}>\mu_{D_{1}, D_{2}}>m .
$$

Moreover,

(a) $\inf \left\{E_{0}(u): u \in M(\mathcal{D}), \beta(u) \in\left[x_{1}, x_{2}\right]\right\}>m$,

(b) $\lim _{\left|x_{1}-x_{2}\right| \rightarrow+\infty} \sup \left\{E_{0}\left(v_{p, \mathcal{D}}[y]\right): y \in \partial B\left(x_{1}, \frac{1}{2}\left|x_{2}-x_{1}\right|\right)\right\}=m$,

(c) $\lim _{\left|x_{1}-x_{2}\right| \rightarrow+\infty} \sup _{y \in \partial B\left(x_{1},\left|x_{1}-x_{2}\right| / 2\right)}\left|\beta\left(v_{p, \mathcal{D}}[y]\right)-y\right|=0$.

Proposition 3.5. Let $a_{1}$ and $a_{2}$ be non-negative functions in $L^{N / 2}\left(\mathbb{R}^{N}\right)$, with $a_{2} \not \equiv 0$, and let $\omega$ be a bounded open set in $\mathbb{R}^{N}$. Define $\mathcal{D}=\mathbb{R}^{N} \backslash\left(\bar{\omega}+x_{1}\right)$ and $a(x)=a_{1}(x-$ $\left.x_{1}\right)+a_{2}\left(x-x_{2}\right), x_{1}, x_{2} \in \mathbb{R}^{N}$; if $a_{1} \not \equiv 0$ or $\omega \neq \emptyset$, then there exists $\mu_{\omega, a_{1}, a_{2}}>m$ such that

$$
\inf _{x_{1}, x_{2} \in \mathbb{R}^{N}} \inf \left\{E_{a}(u): u \in M(\mathcal{D}), \beta(u) \in\left\{x_{1}, x_{2}\right\}\right\}>\mu_{\omega, a_{1}, a_{2}}>m .
$$

Moreover,

(a) $\inf \left\{E_{a}(u): u \in M(\mathcal{D}), \beta(u) \in\left[x_{1}, x_{2}\right]\right\}>m$,

(b) $\lim _{\left|x_{1}-x_{2}\right| \rightarrow+\infty} \sup \left\{E_{a}\left(v_{p, \mathcal{D}}(\cdot-y)\right): y \in \partial B\left(x_{1}, \frac{1}{2}\left|x_{2}-x_{1}\right|\right)\right\}=m$,

(c) $\lim _{\left|x_{1}-x_{2}\right| \rightarrow+\infty} \sup _{y \in \partial B\left(x_{1},\left|x_{2}-x_{1}\right| / 2\right)}\left|\beta\left(v_{p, \mathcal{D}}[y]\right)-y\right|=0$. 
Remark 3.6. From Proposition 3.4 (c) it follows that, for sufficiently large $\left|x_{2}-x_{1}\right|$, there exists $y \in \partial B\left(x_{1}, \frac{1}{2}\left|x_{2}-x_{1}\right|\right)$ such that $\beta\left(v_{p, \mathcal{D}}[y]\right) \in\left[x_{1}, x_{2}\right]$, where $\mathcal{D}=\mathbb{R}^{N} \backslash$ $\bigcup_{i=1}^{2}\left(\bar{D}_{i}+x_{i}\right)$. An analogous assertion follows from Proposition $3.5(\mathrm{c})$.

Proposition 3.7. If $a \in L^{N / 2}\left(\mathbb{R}^{N}\right)$ is a non-negative function, $a \not \equiv 0$, and $\bar{x} \in \mathbb{R}^{N}$, then

(a) $I_{a}:=\inf \left\{E_{a(\cdot-\bar{x})}(u): u \in M\left(\mathbb{R}^{N}\right), \beta_{\bar{x}}(u)=\bar{x}\right\}>m$,

(b) $\lim _{\rho \rightarrow+\infty} \sup \left\{E_{a(\cdot-\bar{x})}\left(\Psi_{\mathbb{R}^{N}, \rho, \bar{x}}[y, 0]\right): y \in \Sigma_{\bar{x}}\right\}=m$.

Remark 3.8. From the symmetry of $v_{p}$, it follows that, for all $\rho>0$ and all $y \in \Sigma_{\bar{x}}$,

$$
\beta_{\bar{x}}\left(\Psi_{\mathbb{R}^{N}, \rho, \bar{x}}[y, 0]\right)=\bar{x}+\theta(\rho|y-\bar{x}|) \frac{y-\bar{x}}{|y-\bar{x}|}, \quad \text { with } \theta(\tau)>0, \forall \tau>0 .
$$

Then, as a consequence of the continuity of $\beta_{\bar{x}}$, for every $\rho>0$ there exists $(\hat{y}, \hat{t}) \in$ $\Sigma_{\bar{x}} \times[0,1]$ such that $\beta_{\bar{x}}\left(\Psi_{\mathbb{R}^{N}, \rho, \bar{x}}[\hat{y}, \hat{t}]\right)=\bar{x}$.

Proposition 3.9. Let $\omega \subset \mathbb{R}^{N}$ be a bounded non-empty open set and $\bar{x} \in \mathbb{R}^{N}$. If $\mathcal{D}=\mathbb{R}^{N} \backslash(\bar{\omega}+\bar{x})$, then

(a) $J_{\omega}:=\inf \left\{E_{0}(u): u \in M(\mathcal{D}), \beta_{\bar{x}}(u)=\bar{x}\right\}>m$,

(b) $\lim _{\rho \rightarrow+\infty} \sup \left\{E_{0}\left(\Psi_{\mathcal{D}, \rho, \bar{x}}[y, 0]\right): y \in \Sigma_{\bar{x}}\right\}=m$,

(c) $\lim _{\rho \rightarrow+\infty} \sup _{y \in \Sigma_{\bar{x}}}\left|\beta_{\bar{x}}\left(\Psi_{\mathcal{D}, \rho, \bar{x}}[y, 0]\right)-(\bar{x}+\chi(\rho(y-\bar{x})))\right|=0$

(see (3.2) for the definition of $\chi$ ).

Remark 3.10. From Proposition 3.9 (c) it follows that, for large $\rho$, there exists $(\hat{y}, \hat{t}) \in \Sigma_{\bar{x}} \times[0,1]$ such that $\beta_{\bar{x}}\left(\Psi_{\mathcal{D}, \rho, \bar{x}}[\hat{y}, \hat{t}]\right)=\bar{x}$.

Now, let us establish what happens when $\omega$ enlarges and $a$ increases. For $\omega \subset \mathbb{R}^{N}$ let us set

$$
D(\omega)=\sup \left\{r \in \mathbb{R}^{+}: B(y, r) \subset \omega \text { for some } y \in \mathbb{R}^{N}\right\} .
$$

Proposition 3.11. Let $\omega \subset \mathbb{R}^{N}$ be a bounded open set, $\bar{x} \in \mathbb{R}^{N}$ and define $\mathcal{D}=$ $\mathbb{R}^{N} \backslash(\bar{\omega}+\bar{x})$. There then exists $\bar{\rho}=\bar{\rho}(\omega)$ such that

$$
\max _{\Sigma_{\bar{x}} \times[0,1]} E_{0}\left(\Psi_{\mathcal{D}, \rho, \bar{x}}[y, t]\right)<2^{1-2 / p} m, \quad \forall \rho>\bar{\rho} .
$$

Moreover, if $B(0, D(\omega)) \subset \bar{\omega}$, then

$$
\lim _{D(\omega) \rightarrow+\infty} J_{\omega}=2^{1-2 / p} m
$$

( $J_{\omega}$ was introduced in Proposition 3.9 (a).)

Inequality (3.19) is [10, Lemma 3.5]; (3.20) is [19, Lemma 3.3]. 
Proposition 3.12. Let $\left(a_{n}\right)_{n}$ be a sequence of non-negative functions in $L^{N / 2}\left(\mathbb{R}^{N}\right)$ that verify condition (1.2), and let $\bar{x} \in \mathbb{R}^{N}$. For every $n \in \mathbb{N}$ there exists $\bar{\rho}=\bar{\rho}\left(a_{n}\right)$ such that

$$
\max _{\Sigma_{\bar{x} \times[0,1]}} E_{a_{n}(\cdot-\bar{x})}\left(\Psi_{\mathbb{R}^{N}, \rho, \bar{x}}[y, t]\right)<2^{1-2 / p} m, \quad \forall \rho>\bar{\rho} .
$$

If

$$
\lim _{n \rightarrow+\infty} a_{n}(x)=+\infty \quad \text { for a.a. } x \in \mathbb{R}^{N}
$$

then

$$
\lim _{n \rightarrow+\infty} I_{a_{n}}=2^{1-2 / p} m
$$

( $I_{a}$ was introduced in Proposition 3.7 (a).)

We can prove (3.21) and (3.22) by arguing as in the proof of [21, Lemmas 2.7 and 3.2], where condition (1.2) has been considered with $\sigma=2$.

\section{A multiplicity result}

Theorem 4.1. Let $h, k \in \mathbb{N}, h+k \neq 0$. Assume that $\Omega$ is a smooth open set in $\mathbb{R}^{N}$, $\Omega \neq \mathbb{R}^{N}$, that satisfies Assumption $(C)$ and that $\left(a_{n}^{1}\right)_{n}, \ldots,\left(a_{n}^{k}\right)_{n}$ are $k$ sequences of non-negative functions in $L^{N / 2}\left(\mathbb{R}^{N}\right)$ verifying condition (1.2) and such that

$$
\lim _{n \rightarrow+\infty} a_{n}^{j}(x)=+\infty \quad \text { for a.a. } x \in \mathbb{R}^{N}, \text { for } j=1, \ldots, k .
$$

There then exist

$$
\begin{aligned}
D_{2}= & D_{2}\left(\omega_{1}\right), \\
D_{3}= & D_{3}\left(\omega_{2}\right), \\
& \vdots \\
D_{h}= & D_{h}\left(\omega_{h-1}\right), \\
\bar{n}_{1}= & \bar{n}_{1}\left(\omega_{h}\right), \\
\bar{n}_{2}= & \bar{n}_{2}\left(a_{n_{1}}^{1}\right), \\
& \vdots \\
& \bar{n}_{k}=\bar{n}_{k}\left(a_{n_{k-1}}^{k-1}\right), \\
\bar{x}_{1}= & \bar{x}_{1}\left(\Omega, \omega_{1}\right), \\
\vdots & \\
\bar{x}_{h}= & \bar{x}_{h}\left(\Omega, \omega_{1}, \bar{x}_{1}, \ldots, \omega_{h-1}, \bar{x}_{h-1}, \omega_{h}\right), \\
\bar{x}_{h+1}= & \bar{x}_{h+1}\left(\mathcal{D}, a_{n_{1}}^{1}\right), \\
& \vdots \\
\bar{x}_{h+k}= & \bar{x}_{h+k}\left(\mathcal{D}, a_{n_{1}}^{1}, \bar{x}_{h+1}, \ldots, a_{n_{k-1}}^{k-1}, \bar{x}_{h+k-1}, a_{n_{k}}^{k}\right),
\end{aligned}
$$


such that problem $P(a, \mathcal{D})$, where $\mathcal{D}=\Omega \backslash \bigcup_{i=1}^{h}\left(\bar{\omega}_{i}+\bar{x}_{i}\right)$, has at least $2(k+h)$ distinct solutions whenever $\omega_{i}$ are open bounded sets in $\mathbb{R}^{N}$ such that $D\left(\omega_{i}\right)>D_{i}$, for $i=$ $2, \ldots, h$, and $a(x)$ has the form

$$
a(x)=\sum_{j=1}^{k} a_{n_{j}}^{j}\left(x-\bar{x}_{h+j}\right),
$$

with $n_{j}>\bar{n}_{j}$, for $j=1, \ldots, k$.

Proof. We assume, in the first steps, that $\Omega=\mathbb{R}^{N}$. Moreover, we can assume that $B\left(0, D\left(\omega_{i}\right)\right) \subset \bar{\omega}_{i}, i=1, \ldots, h$.

Step 1. Let us fix $i \in\{1, \ldots, h\}, x_{i} \in \mathbb{R}^{N}$ and set $\mathcal{D}_{i}=\mathbb{R}^{N} \backslash\left(\bar{\omega}_{i}+x_{i}\right)$.

From Propositions 2.4, 3.9 and 3.11, and taking into account Remark 3.10, it follows that there exists $\bar{\rho}_{i}>0$ such that

$\beta_{x_{i}}\left(\Psi_{\mathcal{D}_{i}, \bar{\rho}_{i}, x_{i}}[y, 0]\right)$ is homotopically equivalent in $\mathbb{R}^{N} \backslash\left\{x_{i}\right\}$ to the identity map on $\Sigma_{x_{i}}$

and

$$
\begin{aligned}
m & <\sup \left\{E_{0}\left(\Psi_{\mathcal{D}_{i}, \bar{\rho}_{i}, x_{i}}[y, 0]\right): y \in \Sigma_{x_{i}}\right\} \\
& <\inf \left\{E_{0}(u): u \in M\left(\mathcal{D}_{i}\right), \beta_{x_{i}}(u)=x_{i}\right\} \\
& \leqslant \sup \left\{E_{0}\left(\Psi_{\mathcal{D}_{i}, \bar{\rho}_{i}, x_{i}}[y, t]\right):(y, t) \in \Sigma_{x_{i}} \times[0,1]\right\} \\
& <2^{1-2 / p} m .
\end{aligned}
$$

Moreover, again by Proposition 3.11, we find that for every $i \in\{1, \ldots, h-1\}$ there exists $D_{i+1}=D_{i+1}\left(\omega_{i}\right)>0$ such that

$$
\begin{aligned}
\sup \left\{E_{0}\left(\Psi_{\mathcal{D}_{i}, \bar{\rho}_{i}, x_{i}}[y, t]\right):(y, t) \in\right. & \left.\Sigma_{x_{i}} \times[0,1]\right\} \\
& <\inf \left\{E_{0}(u): u \in M\left(\mathcal{D}_{i+1}\right), \beta_{x_{i+1}}(u)=x_{i+1}\right\}
\end{aligned}
$$

whenever $D\left(\omega_{i+1}\right)>D_{i+1}$. In the following, $\omega_{i}$ will be considered fixed, for $i=1, \ldots, h$, as chosen in this step.

Step 2. Let us fix $j \in\{1, \ldots, k\}, x_{h+j} \in \mathbb{R}^{N}$ and $n \in \mathbb{N}$. By Propositions 2.4, 3.7, 3.12 and Remark 3.8, we can find $\bar{\rho}_{j}^{n}>0$ such that

$$
\begin{array}{r}
\beta_{x_{h+j}}\left(\Psi_{\mathbb{R}^{N}, \bar{\rho}_{j}^{n}, x_{h+j}}[y, 0]\right) \text { is homotopically equivalent in } \mathbb{R}^{N} \backslash\left\{x_{h+j}\right\} \\
\text { to the identity map on } \Sigma_{x_{h+j}}
\end{array}
$$

and

$$
\begin{aligned}
m & <\sup \left\{E_{a_{n}^{j}\left(\cdot-x_{h+j}\right)}\left(\Psi_{\mathbb{R}^{N}, \bar{\rho}_{j}^{n}, x_{h+j}}[y, 0]\right): y \in \Sigma_{x_{h+j}}\right\} \\
& <\inf \left\{E_{a_{n}^{j}\left(\cdot-x_{h+j}\right)}(u): u \in M\left(\mathbb{R}^{N}\right), \beta_{x_{h+j}}(u)=x_{h+j}\right\} \\
& \leqslant \sup \left\{E_{a_{n}^{j}\left(\cdot-x_{h+j}\right)}\left(\Psi_{\mathbb{R}^{N}, \bar{\rho}_{j}^{n}, x_{h+j}}[y, t]\right):(y, t) \in \Sigma_{x_{h+j}} \times[0,1]\right\} \\
& <2^{1-2 / p} m .
\end{aligned}
$$


Moreover, by Proposition 3.12, we can choose $\bar{n}_{1} \in \mathbb{N}$ such that

$$
\begin{aligned}
\sup \left\{E_{0}\left(\Psi_{\mathcal{D}_{h}, \bar{\rho}_{h}, x_{h}}[y, t]\right):\right. & \left.(y, t) \in \Sigma_{x_{h}} \times[0,1]\right\} \\
& <\inf \left\{E_{a_{n}^{1}\left(-x_{h+1}\right)}(u): u \in M\left(\mathbb{R}^{N}\right), \beta_{x_{h+1}}(u)=x_{h+1}\right\}
\end{aligned}
$$

for every $n>\bar{n}_{1}$ and, fixing $n_{j-1} \in \mathbb{N}$ for $j \in\{2, \ldots, k\}$, there exists $\bar{n}_{j}=\bar{n}_{j}\left(a_{n_{j-i}}^{j-1}\right) \in \mathbb{N}$ such that

$$
\begin{aligned}
\sup \left\{E_{a_{n_{j-1}^{j}-1}\left(-x_{h+j-1)}\right.}(\right. & \left.\left.\Psi_{\mathbb{R}^{N}, \bar{\rho}_{j-1}^{n_{j-1}}, x_{h+j-1}}[y, t]\right):(y, t) \in \Sigma_{x_{h+j-1}} \times[0,1]\right\} \\
& <\inf \left\{E_{a_{n}^{j}\left(-x_{h+j}\right)}(u): u \in M\left(\mathbb{R}^{N}\right), \beta_{x_{h+j}}(u)=x_{h+j}\right\},
\end{aligned}
$$

for every $n>\bar{n}_{j}$. In the following, $n_{j}$ will be considered fixed, for $j=1, \ldots, k$, as chosen in this step. Moreover, we will define $\bar{\rho}_{j}^{n_{j}}=\bar{\rho}_{h+j}$.

Notice that in Steps 1 and $2 \bar{\rho}_{i}, D_{i}$ and $\bar{n}_{i}$ are independent of the translation points $x_{i}$.

Step 3. From Proposition 3.4, with $\omega_{i}$ in place of $D_{i}$ (hence $\mathcal{D}=\mathbb{R}^{N} \backslash \bigcup_{i=1}^{2}\left(\bar{\omega}_{i}+x_{i}\right)$ ), and from Remark 3.6, it follows that there exists $R_{2}=R_{2}\left(\omega_{1}, \omega_{2}\right)$ such that, if $\left|x_{2}-x_{1}\right|>$ $R_{2}$, then we have

$$
\begin{aligned}
m & <\inf \left\{E_{0}(u): u \in M(\mathcal{D}), \beta(u) \in\left[x_{1}, x_{2}\right]\right\} \\
& \leqslant \sup \left\{E_{0}\left(v_{p, \mathcal{D}}[y]\right): y \in \partial B\left(x_{1}, \frac{1}{2}\left|x_{2}-x_{1}\right|\right)\right\} \\
& <\inf \left\{E_{0}(u): u \in M(\mathcal{D}), \beta(u) \in\left\{x_{1}, x_{2}\right\}\right\}
\end{aligned}
$$

and

$$
\sup \left\{E_{0}\left(v_{p, \mathcal{D}}[y]\right): y \in \partial B\left(x_{1}, \frac{1}{2}\left|x_{2}-x_{1}\right|\right)\right\}<\inf \left\{E_{0}(u): u \in M\left(\mathbb{R}^{N} \backslash \bar{\omega}_{1}\right), \beta_{0}(u)=0\right\} .
$$

Moreover, we can choose $R_{2}$ large enough that we also have the following:

$$
\text { the homotopy } \mathcal{H}_{1}: \partial B\left(x_{1}, \frac{1}{2}\left|x_{2}-x_{1}\right|\right) \times[0,1] \rightarrow \mathbb{R}^{N} \backslash\left\{x_{1}, x_{2}\right\}
$$

$$
\text { given by } \mathcal{H}_{1}(y, t)=(1-t) y+t \beta\left(v_{p, \mathcal{D}}[y]\right) \text { is well defined. }
$$

We can apply this procedure recursively for $j=3, \ldots, h$, fixing $x_{i}, i=1, \ldots, j-1$. Proposition 3.4, with $D_{1}=\bigcup_{i=1}^{j-1}\left(\omega_{i}+x_{i}\right)$ and $D_{2}=\omega_{j}$ (hence $\mathcal{D}=\mathbb{R}^{N} \backslash \bigcup_{i=1}^{j}\left(\bar{\omega}_{i}+x_{i}\right)$ ), and Remark 3.6 guarantee the existence of $R_{j}=R_{j}\left(\omega_{1}, \omega_{2}, x_{2}, \ldots, x_{j-1}, \omega_{j}\right)$ such that, for $\left|x_{j}-x_{j-1}\right|>R_{j}$,

$$
\begin{aligned}
m & <\inf \left\{E_{0}(u): u \in M(\mathcal{D}), \beta(u) \in\left[x_{j-1}, x_{j}\right]\right\} \\
& \leqslant \sup \left\{E_{0}\left(v_{p, \mathcal{D}}[y]\right): y \in \partial B\left(x_{j-1}, \frac{1}{2}\left|x_{j}-x_{j-1}\right|\right)\right\} \\
& <\inf \left\{E_{0}(u): u \in M(\mathcal{D}), \beta(u) \in\left\{x_{j-1}, x_{j}\right\}\right\}
\end{aligned}
$$

and

$$
\begin{aligned}
\sup \left\{E_{0}\left(v_{p, \mathcal{D}}[y]\right)\right. & \left.: y \in \partial B\left(x_{j-1}, \frac{1}{2}\left|x_{j}-x_{j-1}\right|\right)\right\} \\
& <\inf \left\{E_{0}(u): u \in M\left(\mathbb{R}^{N} \backslash \bigcup_{i=1}^{j-1}\left(\bar{\omega}_{i}+x_{i}\right)\right), \beta(u) \in\left[x_{j-2}, x_{j-1}\right]\right\} .
\end{aligned}
$$


Moreover, $R_{j}$ can be chosen large enough that we also have the following:

the homotopy $\mathcal{H}_{j-1}: \partial B\left(x_{j-1}, \frac{1}{2}\left|x_{j}-x_{j-1}\right|\right) \times[0,1] \rightarrow \mathbb{R}^{N} \backslash\left\{x_{j-1}, x_{j}\right\}$

given by $\mathcal{H}_{j-1}(y, t)=(1-t) y+t \beta\left(v_{p, \mathcal{D}}[y]\right)$ is well defined. (4.14)

Step 4. Fix $\mathcal{D}=\mathbb{R}^{N} \backslash \bigcup_{i=1}^{h}\left(\bar{\omega}_{i}+x_{i}\right)$ and, for $n \in \mathbb{N}$ and $x_{h+1} \in \mathbb{R}^{N}$, set $a(x)=$ $a_{n}^{1}\left(x-x_{h+1}\right)$.

From Proposition 3.5, with $a_{1} \equiv 0$ and $a_{2}=a_{n}^{1}$, and Remark 3.6, it follows that there exists $R_{h+1}=R_{h+1}(\mathcal{D}, n)>0$ such that, if $\left|x_{h+1}-x_{h}\right|>R_{h+1}$,

$$
\begin{aligned}
m & <\inf \left\{E_{a}(u): u \in M(\mathcal{D}), \beta(u) \in\left[x_{h}, x_{h+1}\right]\right\} \\
& \leqslant \sup \left\{E_{a}\left(v_{p, \mathcal{D}}[y]\right): y \in \partial B\left(x_{h}, \frac{1}{2}\left|x_{h+1}-x_{h}\right|\right)\right\} \\
& <\inf \left\{E_{a}(u): u \in M(\mathcal{D}), \beta(u) \in\left\{x_{h}, x_{h+1}\right\}\right\}
\end{aligned}
$$

and

$$
\begin{aligned}
\sup \left\{E_{a}\left(v_{p, \mathcal{D}}[y]\right): y \in \partial B\left(x_{h}, \frac{1}{2} \mid\right.\right. & \left.\left.x_{h+1}-x_{h} \mid\right)\right\} \\
& <\inf \left\{E_{0}(u): u \in M(\mathcal{D}), \beta(u) \in\left[x_{h-1}, x_{h}\right]\right\} .
\end{aligned}
$$

Moreover, we can choose $R_{h+1}$ large enough that we also have the following:

the homotopy $\mathcal{H}_{h}: \partial B\left(x_{h}, \frac{1}{2}\left|x_{h+1}-x_{h}\right|\right) \times[0,1] \rightarrow \mathbb{R}^{N} \backslash\left\{x_{h}, x_{h+1}\right\}$

given by $\mathcal{H}_{h}(y, t)=(1-t) y+t \beta\left(v_{p, \mathcal{D}}[y]\right)$ is well defined.

Step 5. Fix $\mathcal{D}=\mathbb{R}^{N} \backslash \bigcup_{i=1}^{h}\left(\bar{\omega}_{i}+x_{i}\right)$ and, for $j=2, \ldots, k$, define

$$
a(x)=\sum_{i=1}^{j} a_{n_{i}}^{i}\left(x-x_{h+i}\right),
$$

$n_{i} \in \mathbb{N}$ and $x_{h+i} \in \mathbb{R}^{N}$. Taking into account Proposition 3.5 and Remark 3.6, we see that there exists $R_{h+j}=R_{h+j}\left(\mathcal{D}, n_{1}, x_{h+1}, \ldots, n_{j-1}, x_{h+j-1}, n_{j}\right)$ such that, for $\mid x_{h+j}-$ $x_{h+j-1} \mid>R_{h+j}$,

$$
\begin{aligned}
m & <\inf \left\{E_{a}(u): u \in M(\mathcal{D}), \beta(u) \in\left[x_{h+j-1}, x_{h+j}\right]\right\} \\
& \leqslant \sup \left\{E_{a}\left(v_{p, \mathcal{D}}[y]\right): y \in \partial B\left(x_{h+j-1}, \frac{1}{2}\left|x_{h+j}-x_{h+j-1}\right|\right)\right\} \\
& <\inf \left\{E_{a}(u): u \in M(\mathcal{D}), \beta(u) \in\left\{x_{h+j-1}, x_{h+j}\right\}\right\}
\end{aligned}
$$

and

$$
\begin{aligned}
\sup \left\{E_{a}\left(v_{p, \mathcal{D}}[y]\right): y \in \partial\right. & \left.\left(x_{h+j-1}, \frac{1}{2}\left|x_{h+j}-x_{h+j-1}\right|\right)\right\} \\
& <\inf \left\{E_{a}(u): u \in M(\mathcal{D}), \beta(u) \in\left[x_{h+j-2}, x_{h+j-1}\right]\right\} .
\end{aligned}
$$

Moreover, $R_{h+j}$ can be chosen large enough that we also have:

the homotopy $\mathcal{H}_{h+j-1}: \partial B\left(x_{h+j-1}, \frac{1}{2}\left|x_{h+j}-x_{h+j-1}\right|\right) \times[0,1] \rightarrow \mathbb{R}^{N} \backslash\left\{x_{h+j-1}, x_{h+j}\right\}$ given by $\mathcal{H}_{h+j-1}(y, t)=(1-t) y+t \beta\left(v_{p, \mathcal{D}}[y]\right)$ is well defined. $\quad(4.20)$ 
Step 6. First, set $\mathcal{D}_{1,2}=\mathbb{R}^{N} \backslash \bigcup_{i=1}^{2}\left(\bar{\omega}_{i}+x_{i}\right)$. From (2.6), (2.7) it follows that, for $i=1,2$,

$\lim _{\left|x_{2}-x_{1}\right| \rightarrow+\infty} \max \left\{E_{0}\left(\Psi_{\mathcal{D}_{1,2}, \bar{\rho}_{i}, x_{i}}[y, t]\right):(y, t) \in K\right\}=\max \left\{E_{0}\left(\Psi_{\mathcal{D}_{i}, \bar{\rho}_{i}, x_{i}}[y, t]\right):(y, t) \in K\right\}$,

for every compact subset $K$ in $\Sigma_{x_{i}} \times[0,1]$. Moreover,

$$
\lim _{\left|x_{2}-x_{1}\right| \rightarrow+\infty} \beta_{x_{i}}\left(\Psi_{\mathcal{D}_{1,2}, \bar{\rho}_{i}, x_{i}}[y, 0]\right)=\beta_{x_{i}}\left(\Psi_{\mathcal{D}_{i}, \bar{\rho}_{i}, x_{i}}[y, 0]\right) \quad \text { uniformly in } \Sigma_{x_{i}}, i=1,2 .
$$

Hence, in particular, for $\left|x_{2}-x_{1}\right|$ sufficiently large, (4.2) holds with $\mathcal{D}_{1,2}$ in place of $\mathcal{D}_{i}$, so

$$
\exists\left(\bar{z}_{i}, \bar{t}_{i}\right) \in \Sigma_{x_{i}} \times[0,1] \quad \text { such that } \beta_{x_{i}}\left(\Psi_{\mathcal{D}_{1,2}, \bar{\rho}_{i}, x_{i}}\left[\bar{z}_{i}, \bar{t}_{i}\right]\right)=x_{i} .
$$

Now observe that

$$
\begin{aligned}
\inf \left\{E_{0}(u): u \in M\left(\mathcal{D}_{i}\right),\right. & \left.\beta_{x_{i}}(u)=x_{i}\right\} \\
& \leqslant \inf \left\{E_{0}(u): u \in M\left(\mathcal{D}_{1,2}\right), \beta_{x_{i}}(u)=x_{i}\right\}, \quad i=1,2 .
\end{aligned}
$$

From (4.21), (4.23) and (4.24) it follows that, if $\left|x_{2}-x_{1}\right|$ is sufficiently large, (4.3) holds with $\mathcal{D}_{1,2}$ in place of $\mathcal{D}_{i}, i=1,2$, and (4.4) holds with $i=1$ and $\mathcal{D}_{1}=\mathcal{D}_{2}=\mathcal{D}_{1,2}$.

Furthermore, we can also take $\left|x_{2}-x_{1}\right|$ to be sufficiently large to get (4.9)-(4.11).

Now, set $\mathcal{D}_{1,2,3}=\mathbb{R}^{N} \backslash \bigcup_{i=1}^{3}\left(\bar{\omega}_{i}+x_{i}\right)$, with $x_{1}$ and $x_{2}$ fixed as in the previous claim. We find that

$$
\begin{aligned}
\lim _{\left|x_{3}-x_{2}\right| \rightarrow+\infty} \max \left\{E_{0}\left(\Psi_{\mathcal{D}_{1,2,3}, \bar{\rho}_{3}, x_{3}}[y, t]\right)\right. & :(y, t) \in K\} \\
& =\max \left\{E_{0}\left(\Psi_{\mathcal{D}_{3}, \bar{\rho}_{3}, x_{3}}[y, t]\right):(y, t) \in K\right\},
\end{aligned}
$$

for every compact subset $K$ in $\Sigma_{x_{3}} \times[0,1]$. Moreover,

$$
\lim _{\left|x_{3}\right| \rightarrow+\infty} \beta_{x_{3}}\left(\Psi_{\mathcal{D}_{1,2,3}, \bar{\rho}_{3}, x_{3}}[y, 0]\right)=\beta_{x_{3}}\left(\Psi_{\mathcal{D}_{3}, \bar{\rho}_{3}, x_{3}}[y, 0]\right) \quad \text { uniformly in } \Sigma_{x_{3}},
$$

so, for $\left|x_{3}\right|$ sufficiently large, (4.2) holds for $i=3$, with $\mathcal{D}_{1,2,3}$ in place of $\mathcal{D}_{3}$ and

$$
\exists\left(\bar{z}_{3}, \bar{t}_{3}\right) \in \Sigma_{x_{3}} \times[0,1] \quad \text { such that } \beta_{x_{3}}\left(\Psi_{\mathcal{D}_{1,2,3}, \bar{\rho}_{3}, x_{3}}\left[\bar{z}_{3}, \bar{t}_{3}\right]\right)=x_{3} .
$$

Observe also that

$$
\inf \left\{E_{0}(u): u \in M\left(\mathcal{D}_{3}\right), \beta_{x_{3}}(u)=x_{3}\right\} \leqslant \inf \left\{E_{0}(u): u \in M\left(\mathcal{D}_{1,2,3}\right), \beta_{x_{3}}(u)=x_{3}\right\} .
$$

From (4.25), (4.27) and (4.28) it follows that, for $\left|x_{3}\right|$ large enough, (4.3) holds, for $i=3$, with $\mathcal{D}_{1,2,3}$ in place of $\mathcal{D}_{3}$.

We have, for $i=1,2$,

$$
\lim _{\left|x_{3}\right| \rightarrow+\infty} \max \left\{E_{0}\left(\Psi_{\mathcal{D}_{1,2,3}, \bar{\rho}_{i}, x_{i}}[y, t]\right):(y, t) \in K\right\}=\max \left\{E_{0}\left(\Psi_{\mathcal{D}_{1,2}, \bar{\rho}_{i}, x_{i}}[y, t]\right):(y, t) \in K\right\},
$$


for every compact subset $K$ in $\Sigma_{x_{i}} \times[0,1]$. Furthermore,

$$
\lim _{\left|x_{3}\right| \rightarrow+\infty} \beta_{x_{i}}\left(\Psi_{\mathcal{D}_{1,2,3}, \bar{\rho}_{i}, x_{i}}[y, 0]\right)=\beta_{x_{i}}\left(\Psi_{\mathcal{D}_{1,2}, \bar{\rho}_{i}, x_{i}}[y, 0]\right) \quad \text { uniformly in } \Sigma_{x_{i}} .
$$

Hence, for $\left|x_{3}\right|$ sufficiently large, (4.2) holds with $\mathcal{D}_{1,2,3}$ in place of $\mathcal{D}_{i}$, for $i=1,2$, and

$$
\exists\left(\bar{z}_{i}, \bar{t}_{i}\right) \in \Sigma_{x_{i}} \times[0,1] \quad \text { such that } \beta_{x_{i}}\left(\Psi_{\mathcal{D}_{1,2,3}, \bar{\rho}_{i}, x_{i}}\left[\bar{z}_{i}, \bar{t}_{i}\right]\right)=x_{i} .
$$

Now, observe that

$$
\begin{aligned}
& \inf \left\{E_{0}(u): u \in M\left(\mathcal{D}_{1,2}\right), \beta_{x_{i}}(u)=x_{i}\right\} \\
& \quad \leqslant \inf \left\{E_{0}(u): u \in M\left(\mathcal{D}_{1,2,3}\right), \beta_{x_{i}}(u)=x_{i}\right\} \quad \text { for } i=1,2 .
\end{aligned}
$$

So (4.29), (4.31) and (4.32) imply that, for sufficiently large $\left|x_{3}\right|$, (4.3) also holds, for $i=1,2$, with $\mathcal{D}_{1,2,3}$ in place of $\mathcal{D}_{i}$.

Moreover, taking into account (4.25), (4.29) and (4.28), (4.32), we get (4.4) with $i=1,2$ and $\mathcal{D}_{i}=\mathcal{D}_{i+1}=\mathcal{D}_{1,2,3}$.

We have

$$
\begin{aligned}
\lim _{\left|x_{3}\right| \rightarrow+\infty} \max \left\{E_{0}\left(v_{p, \mathcal{D}_{1,2,3}}[y]\right): y\right. & \left.\in \partial B\left(x_{1}, \frac{1}{2}\left|x_{2}-x_{1}\right|\right)\right\} \\
= & \max \left\{E_{0}\left(v_{p, \mathcal{D}_{1,2}}[y]\right): y \in \partial B\left(x_{1}, \frac{1}{2}\left|x_{2}-x_{1}\right|\right)\right\}
\end{aligned}
$$

and

$$
\begin{aligned}
\inf \left\{E_{0}(u): u \in\right. & \left.M\left(\mathcal{D}_{1,2}\right), \beta(u) \in\left\{x_{1}, x_{2}\right\}\right\} \\
& \leqslant \inf \left\{E_{0}(u): u \in M\left(\mathcal{D}_{1,2,3}\right), \beta(u) \in\left\{x_{1}, x_{2}\right\}\right\}, \quad \forall x_{3} \in \mathbb{R}^{N} .
\end{aligned}
$$

Furthermore,

$$
\lim _{\left|x_{3}\right| \rightarrow+\infty} \beta\left(v_{p, \mathcal{D}_{1,2,3}}[y]\right)=\beta\left(v_{p, \mathcal{D}_{1,2}}[y]\right) \quad \text { uniformly in } \partial B\left(x_{1}, \frac{1}{2}\left|x_{2}-x_{1}\right|\right),
$$

so, for $\left|x_{3}\right|$ sufficiently large, (4.11) holds with $\mathcal{D}_{1,2,3}$ in place of $\mathcal{D}$ and, in particular,

$$
\exists y_{1,2} \in \partial B\left(x_{1}, \frac{1}{2}\left|x_{2}-x_{1}\right|\right) \quad \text { such that } \beta\left(v_{p, \mathcal{D}_{1,2,3}}\left[y_{1,2}\right]\right) \in\left[x_{1}, x_{2}\right] .
$$

Then, from (4.33)-(4.36) it follows that, for sufficiently large $\left|x_{3}\right|,(4.9)-(4.11)$ hold with $\mathcal{D}_{1,2,3}$ in place of $\mathcal{D}$.

We can also take $\left|x_{3}\right|$ sufficiently large to verify (4.12)-(4.14) with $j=3$.

Iterating these arguments, we get (4.2), (4.3) with $\mathbb{R}^{N} \backslash \bigcup_{i=1}^{h}\left(\bar{\omega}_{i}+x_{i}\right)$ in place of $\mathcal{D}_{i}$, $i=1, \ldots, h,(4.4)$ with

$$
\mathcal{D}_{i}=\mathcal{D}_{i+1}=\mathbb{R}^{N} \backslash \bigcup_{i=1}^{h}\left(\bar{\omega}_{i}+x_{i}\right), \quad i=1, \ldots, h-1,
$$

and (4.9)-(4.14) with $\mathbb{R}^{N} \backslash \bigcup_{i=1}^{h}\left(\bar{\omega}_{i}+x_{i}\right)$ in place of $\mathcal{D}$. 
Now, fix $\mathcal{D}=\mathbb{R}^{N} \backslash \bigcup_{i=1}^{h}\left(\bar{\omega}_{i}+x_{i}\right)$.

Notice that, arguing as for (3.16), for every $a \in L^{N / 2}\left(\mathbb{R}^{N}\right)$ we obtain

$\lim _{|z| \rightarrow+\infty} E_{a(\cdot-z)}(w)=E_{0}(w) \quad$ uniformly in $w \in K, K \subset H_{0}^{1}(\mathcal{D})$ a compact set.

Moreover, for $a(x)=a_{n_{1}}^{1}\left(x-x_{h+1}\right), x_{h+1} \in \mathbb{R}^{N}$,

$$
\begin{aligned}
\inf \left\{E_{0}(u):\right. & \left.u \in M(\mathcal{D}), \beta_{x_{i}}(u)=x_{i}\right\} \\
& \leqslant \inf \left\{E_{a}(u): u \in M(\mathcal{D}), \beta_{x_{i}}(u)=x_{i}\right\}, \quad i=1, \ldots, h,
\end{aligned}
$$

and

$$
\begin{aligned}
\inf \left\{E_{0}(u):\right. & \left.u \in M(\mathcal{D}), \beta(u) \in\left\{x_{i}, x_{i+1}\right\}\right\} \\
& \leqslant \inf \left\{E_{a}(u): u \in M(\mathcal{D}), \beta(u) \in\left\{x_{i}, x_{i+1}\right\}\right\}, \quad i=1, \ldots, h-1 .
\end{aligned}
$$

Hence, we can choose $\left|x_{h+1}\right|$ sufficiently large in such a way that not only do (4.15)(4.17) hold, but also (4.3), (4.4), (4.9), (4.10) and (4.12), (4.13) hold, on the domain $\mathcal{D}=\mathbb{R}^{N} \backslash \bigcup_{i=1}^{h}\left(\bar{\omega}_{i}+x_{i}\right)$, with the functional $E_{a}$ in place of $E_{0}$.

Notice that from (2.6) and (2.7) it follows, for every $a \in L^{N / 2}\left(\mathbb{R}^{N}\right)$, that, for every compact set $K \subset \Sigma_{0} \times[0,1]$ and for every $\rho>0$,

$$
\lim _{|z| \rightarrow+\infty} E_{a(\cdot-z)}\left(\Psi_{\mathcal{D}, \rho, z}[y+z, t]\right)=E_{a}\left(\Psi_{\mathbb{R}^{N}, \rho, 0}[y, t]\right) \quad \text { uniformly in } K .
$$

Moreover,

$$
\lim _{|z| \rightarrow+\infty} \sup _{y \in \Sigma_{0}}\left|\left(\beta_{z}\left(\Psi_{\mathcal{D}, \rho, z}[y+z, 0]\right)-z\right)-\beta_{0}\left(\Psi_{\mathbb{R}^{N}, \rho, 0}[y, 0]\right)\right|=0 .
$$

So, also taking into account relations (4.37), (4.40), (4.41) and arguing for the bumps $a_{n_{i}}^{i}\left(x-x_{h+i}\right), i=1, \ldots, k$, as we have done for the holes $\left(\bar{\omega}_{i}+x_{i}\right), i=1, \ldots, h$, we can choose recursively the 'centre' of the bumps $x_{h+i}$ in such a way that the inequalities stated in Steps 1-5 hold with $\mathcal{D}=\mathbb{R}^{N} \backslash \bigcup_{i=1}^{h}\left(\bar{\omega}_{i}+x_{i}\right)$ and $E_{a}$ in place of $E_{0}$, where $a(x)=\sum_{i=1}^{k} a_{n_{i}}^{i}\left(x-x_{h+i}\right)$. Namely, we obtain, for $i=1, \ldots, h+k$,

$$
\begin{aligned}
m & <\sup \left\{E_{a}\left(\Psi_{\mathcal{D}, \bar{\rho}_{i}, x_{i}}[y, 0]\right): y \in \Sigma_{x_{i}}\right\} \\
& <b_{1, i}:=\inf \left\{E_{a}(u): u \in M(\mathcal{D}), \beta_{x_{i}}(u)=x_{i}\right\} \\
& \leqslant b_{2, i}:=\sup \left\{E_{a}\left(\Psi_{\mathcal{D}, \bar{\rho}_{i}, x_{i}}[y, t]\right):(y, t) \in \Sigma_{x_{i}} \times[0,1]\right\}<2^{1-2 / p} m,
\end{aligned}
$$

$\beta_{x_{i}}\left(\Psi_{\mathcal{D}, \bar{\rho}_{i}, x_{i}}[y, 0]\right)$ is homotopically equivalent in $\mathbb{R}^{N} \backslash\left\{x_{i}\right\}$ to the identity map on $\Sigma_{x_{i}}$ and, for $i=1, \ldots, h+k-1$,

$$
\sup \left\{E_{a}\left(\Psi_{\mathcal{D}, \bar{\rho}_{i}, x_{i}}[y, t]\right):(y, t) \in \Sigma_{x_{i}} \times[0,1]\right\}<\inf \left\{E_{a}(u): u \in M(\mathcal{D}), \beta_{x_{i+1}}(u)=x_{i+1}\right\} .
$$


Moreover, for $i=1, \ldots, h+k-1$,

$$
\begin{aligned}
m & <d_{1, i}:=\inf \left\{E_{a}(u): u \in M(\mathcal{D}), \beta(u) \in\left[x_{i}, x_{i+1}\right]\right\} \\
& \leqslant d_{2, i}:=\sup \left\{E_{a}\left(v_{p, \mathcal{D}}[y]\right): y \in \partial B\left(x_{i}, \frac{1}{2}\left|x_{i+1}-x_{i}\right|\right)\right\} \\
& <\inf \left\{E_{a}(u): u \in M(\mathcal{D}), \beta(u) \in\left\{x_{i}, x_{i+1}\right\}\right\},
\end{aligned}
$$

the homotopy $\mathcal{H}_{i}: \partial B\left(x_{i}, \frac{1}{2}\left|x_{i+1}-x_{i}\right|\right) \times[0,1] \rightarrow \mathbb{R}^{N} \backslash\left\{x_{i}, x_{i+1}\right\}$

given by $\mathcal{H}_{i}(y, t)=(1-t) y+t \beta\left(v_{p, \mathcal{D}}[y]\right)$ is well defined.

Furthermore,

$$
\begin{aligned}
\sup \left\{E_{a}\left(v_{p, \mathcal{D}}[y]\right): y \in \partial B\right. & \left.\left(x_{1}, \frac{1}{2}\left|x_{2}-x_{1}\right|\right)\right\} \\
& <\inf \left\{E_{a}(u): u \in M(\mathcal{D}), \beta_{x_{1}}(u)=x_{1}\right\}<2^{1-2 / p} m
\end{aligned}
$$

and, for $i=2, \ldots, h+k-1$,

$$
\begin{aligned}
\sup \left\{E_{a}\left(v_{p, \mathcal{D}}[y]\right): y \in\right. & \left.\partial B\left(x_{i}, \frac{1}{2}\left|x_{i+1}-x_{i}\right|\right)\right\} \\
& <\inf \left\{E_{a}(u): u \in M(\mathcal{D}), \beta(u) \in\left[x_{i-1}, x_{i}\right]\right\}<2^{1-2 / p} m .
\end{aligned}
$$

Step 7. By Assumption (C), we can now consider a sequence of points $z_{n} \in \Omega$ such that $B\left(z_{n}, n\right) \subset \Omega$. Fix

$$
D=\bigcup_{j=1}^{h}\left(\bar{\omega}_{j}+x_{j}\right) \quad \text { and } \quad a(x)=\sum_{j=1}^{k} a_{n_{j}}^{j}\left(x-x_{h+j}\right) .
$$

For $i=1, \ldots, h+k$ we have

$$
\begin{aligned}
\lim _{n \rightarrow+\infty} \sup \left\{\mid E_{a\left(\cdot-z_{n}\right)}\left(\Psi_{\mathbb{R}^{N} \backslash\left(D+z_{n}\right), \bar{\rho}_{i}, x_{i}+z_{n}}\left[y+z_{n}, t\right]\right)\right. & \\
& \left.-E_{a\left(\cdot-z_{n}\right)}\left(\Psi_{\Omega \backslash\left(D+z_{n}\right), \bar{\rho}_{i}, x_{i}+z_{n}}\left[y+z_{n}, t\right]\right) \mid:(y, t) \in K\right\}=0,
\end{aligned}
$$

$K$ compact in $\Sigma_{x_{i}} \times[0,1]$

$\lim _{n \rightarrow+\infty} \sup _{y \in \Sigma_{x_{i}+z_{n}}}\left|\beta_{x_{i}+z_{n}}\left(\Psi_{\mathbb{R}^{N} \backslash\left(D+z_{n}\right), \bar{\rho}_{i}, x_{i}+z_{n}}[y, 0]\right)-\beta_{x_{i}+z_{n}}\left(\Psi_{\Omega \backslash\left(D+z_{n}\right), \bar{\rho}_{i}, x_{i}+z_{n}}[y, 0]\right)\right|=0$,

$\inf \left\{E_{a\left(\cdot-z_{n}\right)}(u): u \in M\left(\mathbb{R}^{N} \backslash\left(D+z_{n}\right)\right), \beta_{x_{i}+z_{n}}(u)=x_{i}+z_{n}\right\}$

$$
\leqslant \inf \left\{E_{a\left(-z_{n}\right)}(u): u \in M\left(\Omega \backslash\left(D+z_{n}\right)\right), \beta_{x_{i}+z_{n}}(u)=x_{i}+z_{n}\right\}, \quad \forall n \in \mathbb{N} .
$$

Moreover, for every compact $K$ in $\mathbb{R}^{N}$,

$$
\begin{gathered}
\lim _{n \rightarrow+\infty} \sup _{y \in K}\left|E_{a\left(\cdot-z_{n}\right)}\left(v_{p, \mathbb{R}^{N} \backslash\left(D+z_{n}\right)}\left[y+z_{n}\right]\right)-E_{a\left(\cdot-z_{n}\right)}\left(v_{p, \Omega \backslash\left(D+z_{n}\right)}\left[y+z_{n}\right]\right)\right|=0, \\
\lim _{n \rightarrow+\infty} \sup _{y \in K}\left|\beta\left(v_{p, \mathbb{R}^{N} \backslash\left(D+z_{n}\right)}\left[y+z_{n}\right]\right)-\beta\left(v_{p, \Omega \backslash\left(D+z_{n}\right)}\left[y+z_{n}\right]\right)\right|=0, \\
\inf \left\{E_{a\left(\cdot-z_{n}\right)}(u): u \in M\left(\mathbb{R}^{N} \backslash\left(D+z_{n}\right)\right), \beta(u) \in K+z_{n}\right\} \\
\leqslant \inf \left\{E_{a\left(\cdot-z_{n}\right)}(u): u \in M\left(\Omega \backslash\left(D+z_{n}\right)\right), \beta(u) \in K+z_{n}\right\}, \quad \forall n \in \mathbb{N} .
\end{gathered}
$$


From (4.49)-(4.54) it follows that (4.42)-(4.48) hold, for $n$ large, with

$$
\mathcal{D}=\Omega \backslash \bigcup_{i=1}^{h}\left(\bar{\omega}_{i}+\left(x_{i}+z_{n}\right)\right), \quad a(x)=\sum_{i=1}^{k} a_{n_{i}}^{i}\left(x-\left(x_{h+i}+z_{n}\right)\right)
$$

and setting

$$
\bar{x}_{i}:=x_{i}+z_{n}, \quad i=1, \ldots, h+k,
$$

in place of $x_{i}$. Furthermore, by Propositions 3.1, 3.2 and Remark 3.3, $n$ can be chosen to be large enough to also have

$$
\begin{aligned}
m & <d_{1, h+k}:=\inf \left\{E_{a}(u): u \in M(\mathcal{D}), \beta(u) \in\left[0, z_{n}\right]\right\} \\
& \leqslant d_{2, h+k}:=\sup \left\{E_{a}\left(v_{p, \mathcal{D}}\right): y \in \partial B\left(z_{n}, \frac{1}{2} n\right)\right\} \\
& <\inf \left\{E_{a}(u): u \in M(\mathcal{D}), \beta(u) \in\left\{0, z_{n}\right\}\right\}
\end{aligned}
$$

the homotopy $\mathcal{H}_{h+k}: \partial B\left(z_{n}, \frac{1}{2} n\right) \times[0,1] \rightarrow \mathbb{R}^{N} \backslash\left\{0, z_{n}\right\}$

given by $\mathcal{H}_{h+k}(y, t)=(1-t) y+t \beta\left(v_{p, \mathcal{D}}[y]\right)$ is well defined

and, moreover,

$$
\begin{aligned}
m & <\sup \left\{E_{a}\left(v_{p, \mathcal{D}}\right): y \in \partial B\left(z_{n}, \frac{1}{2} n\right)\right\} \\
& <\inf \left\{E_{a}(u): u \in M(\mathcal{D}), \beta(u) \in\left[\bar{x}_{h+k-1}, \bar{x}_{h+k}\right]\right\}<2^{1-2 / p} m .
\end{aligned}
$$

Step 8. We now prove Theorem 4.1 with $D_{2}, \ldots, D_{h}$ as in Step $1, \bar{n}_{1}, \ldots, \bar{n}_{k}$ as in Step 2 and $\bar{x}_{1}, \ldots, \bar{x}_{h+k}$ as in Step 7. Henceforth,

$$
\mathcal{D}=\Omega \backslash \bigcup_{i=1}^{h}\left(\bar{\omega}_{i}+\bar{x}_{i}\right) \quad \text { and } \quad a(x)=\sum_{i=1}^{k} a_{n_{i}}^{i}\left(x-\bar{x}_{h+i}\right) .
$$

We denote the sublevels of $E_{a}$ in $M(\mathcal{D})$ by

$$
E_{a}^{c}=\left\{u \in M(\mathcal{D}): E_{a}(u) \leqslant c\right\}, \quad c \in \mathbb{R} .
$$

For $i=1, \ldots, h+k$, we claim that there exists a critical value $c_{i} \in\left[b_{1, i}, b_{2, i}\right]$ (see (4.42), with $a$ and $\mathcal{D}$ as in (4.59) and $\bar{x}_{i}$ in place of $x_{i}$ ). Assume, by contradiction, that such a critical value does not exist. Then, taking into account Proposition 2.2, standard arguments (see [23], for example) show that there exist $\eta_{i}>0$ and a continuous map

$$
\mathcal{G}_{i}: E_{a}^{b_{2}, i} \rightarrow E_{a}^{b_{1, i}-\eta_{i}}
$$

such that

$$
\mathcal{G}_{i}(u)=u, \quad \forall u \in E_{a}^{b_{1, i}-\eta_{i}} .
$$

Moreover, by (4.42), $\eta_{i}$ can be chosen small enough to have

$$
b_{1, i}-\eta_{i}>\sup \left\{E_{a}\left(\Psi_{\mathcal{D}, \bar{\rho}_{i}, \bar{x}_{i}}[y, 0]\right): y \in \Sigma_{x_{i}}\right\} .
$$


If we identify $B\left(\bar{x}_{i}, 1\right)$ with $\Sigma_{\bar{x}_{i}} \times[0,1]$, by polar coordinates (with the points of the form $(y, 1), y \in \Sigma_{\bar{x}_{i}}$, corresponding to $\bar{x}_{i}$ in $\left.B\left(\bar{x}_{i}, 1\right)\right)$, then, by (4.42), (4.60), (4.61) and (4.43), the map $g_{i}: B\left(\bar{x}_{i}, 1\right) \rightarrow \mathbb{R}^{N}$ given by

$$
g_{i}(y, t)=\beta_{x_{i}}\left(\mathcal{G}_{i}\left(\Psi_{\mathcal{D}, \bar{\rho}_{i}, \bar{x}_{i}}[y, t]\right)\right)
$$

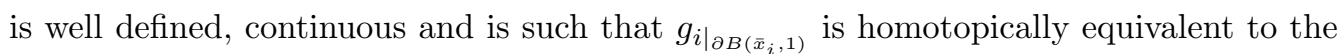
identity in $\mathbb{R}^{N} \backslash\left\{\bar{x}_{i}\right\}$. Hence, there exists $\left(\bar{y}_{i}, \bar{t}_{i}\right) \in \Sigma_{\bar{x}_{i}} \times[0,1]$ such that $g_{i}\left(\bar{y}_{i}, \bar{t}_{i}\right)=\bar{x}_{i}$, i.e. $\beta_{x_{i}}\left(\mathcal{G}_{i}\left(\Psi_{\mathcal{D}, \bar{\rho}_{i}, \bar{x}_{i}}[y, t]\right)\right)=\bar{x}_{i}$, that is in contradiction to $(4.60)$ and proves our claim.

Our next goal is to show that for every $i=1, \ldots, h+k-1$ there exists a critical value $c_{i, i+1} \in\left[d_{1, i}, d_{2, i}\right]$ (see (4.45) with $a$ and $\mathcal{D}$ as in (4.59) and $\bar{x}_{i}$ in place of $x_{i}$ ). Observe that $\left[d_{1, i}, d_{2, i}\right] \subset\left(m, 2^{1-2 / p} m\right)$ by (4.45), (4.47) and (4.48), and assume, by contradiction, that such a critical point does not exist. Then, taking into account Proposition 2.2, we see that there exist $\eta_{i, i+1}>0$ and a continuous deformation $\mathcal{F}_{i}: E_{a}^{d_{2, i}} \times[0,1] \rightarrow E_{a}^{d_{2, i}}$ such that

$$
\mathcal{F}_{i}(u, 0)=u, \forall u \in E_{a}^{d_{2, i}}, \quad \text { and } \quad \mathcal{F}_{i}(u, 1) \subset E_{a}^{d_{1, i}-\eta_{i, i+1}}, \forall u \in E_{a}^{d_{2, i}} .
$$

Now, let us define $f_{i}: \partial B\left(\bar{x}_{i}, \frac{1}{2}\left|\bar{x}_{i+1}-\bar{x}_{i}\right|\right) \times[0,1] \rightarrow \mathbb{R}^{N} \backslash\left\{\bar{x}_{i}, \bar{x}_{i+1}\right\}$ by

$$
f_{i}(y, t)= \begin{cases}\mathcal{H}_{i}(y, 2 t) & \text { if } t \in\left[0, \frac{1}{2}\right], \\ \beta\left(\mathcal{F}_{i}\left(v_{p, \mathcal{D}}[y], 2 t-1\right)\right) & \text { if } t \in\left[\frac{1}{2}, 1\right] .\end{cases}
$$

By (4.46), (4.62) and (4.45), $f_{i}$ is a well-defined continuous deformation that verifies

$$
\begin{aligned}
f_{i}(y, 0) & =y, & \forall y & \in \partial B\left(\bar{x}_{i}, \frac{1}{2}\left|\bar{x}_{i+1}-\bar{x}_{i}\right|\right), \\
f_{i}(y, 1) & \cap\left[\bar{x}_{i}, \bar{x}_{i+1}\right]=\emptyset, & \forall y & \in \partial B\left(\bar{x}_{i}, \frac{1}{2}\left|\bar{x}_{i+1}-\bar{x}_{i}\right|\right), \\
f_{i}(y, t) & \notin\left\{\bar{x}_{i}, \bar{x}_{i+1}\right\}, & \forall(y, t) & \in \partial B\left(\bar{x}_{i}, \frac{1}{2}\left|\bar{x}_{i+1}-\bar{x}_{i}\right|\right) \times[0,1],
\end{aligned}
$$

which is impossible. So the existence of the critical value $c_{i, i+1}$ is proved.

By using relations (4.56)-(4.58) and exactly the same argument developed to prove the existence of the critical values $c_{i, i+1}, i=1, \ldots, h+k-1$, we can find a critical value $c_{h+k, h+k+1} \in\left[d_{1, h+k}, d_{2, h+k}\right]$.

To summarize: from (4.44), (4.47), (4.48) and (4.58) it follows that the critical levels $c_{i}, c_{i, i+1}$, we have found for $i=1, \ldots, h+k$, verify

$$
m<c_{h+k, h+k+1}<c_{h+k-1, h+k}<\cdots<c_{1,2}<c_{1}<\cdots<c_{h+k}<2^{1-2 / p} m .
$$

Hence, they actually give rise to distinct critical points for the functional $E_{a}$ constrained on $M(\mathcal{D})$. These critical points are positive functions, by (4.64), Proposition 2.3 and the maximum principle, so they provide $2(h+k)$ distinct solutions to problem $P(a, \mathcal{D})$. 


\section{Final remarks}

In the proof of Theorem 4.1, $h$ critical levels are related to the $\omega_{i}, k$ to the $a_{i}, h+k-1$ to the interactions between the holes in the domain and the bump in the potential, and another critical level comes from the action of the boundary of $\Omega$. In order to distinguish the critical levels we have found (hence the solutions), a crucial role has been played by the asymptotic behaviour stated in Proposition 3.2 (a), in Propositions 3.4 (b) and 3.5, and in (3.20), (3.22). The application of these results in the proof of Theorem 4.1 shows that if $u_{\omega_{i}}$ are the solutions of $P(a, \mathcal{D})$ related to $\omega_{i}, u_{a_{n_{i}}^{i}}$ are the solutions related to $a_{n_{i}}^{i}$, and $u_{i, i+1}$ are the solutions given by the interactions, then the following energy estimates hold:

$$
\begin{array}{rlrl}
\lim _{D\left(\omega_{i}\right) \rightarrow+\infty} E_{a}\left(\frac{u_{\omega_{i}}}{\left|u_{\omega_{i}}\right|_{L^{p}}}\right) & =2^{1-2 / p} m, \quad i=1, \ldots, h, \\
\lim _{\bar{n}_{i} \rightarrow+\infty} E_{a}\left(\frac{u_{a_{n_{i}}^{i}}}{\left|u_{a_{n_{i}}^{i}}\right|_{L^{p}}}\right) & =2^{1-2 / p} m, \quad i=1, \ldots, k, \\
\lim _{\left|\bar{x}_{i+1}-\bar{x}_{i}\right| \rightarrow+\infty} E_{a}\left(\frac{u_{i, i+1}}{\left|u_{i, i+1}\right|_{L^{p}}}\right) & =m, & \\
\lim _{n \rightarrow+\infty} E_{a}\left(\frac{u_{h+k, h+k+1}}{\left|u_{h+k, h+k+1}\right|_{L^{p}}}\right) & =m
\end{array}
$$

(see (4.56) in Step 7 of the proof of Theorem 4.1 for the dependence on $n$ of the solution $\left.u_{h+k, h+k+1}\right)$. In fact, (5.1) and (5.2) follow from (4.42), (5.3) follows from (4.45), and (5.4) follows from (4.56).

A natural question is what happens if the 'holes' $\omega_{i}$ shrink, instead of enlarging, and the bumps $a^{i}$ vanish, instead of increasing. To analyse this situation, arguing as in the proof of [5, Theorem A.1] we can obtain the following asymptotic estimate:

$$
\lim _{\operatorname{cap} \bar{\omega} \rightarrow 0} \sup \left\{E_{0}\left(v_{p, \mathbb{R}^{N} \backslash \bar{\omega}}[y]\right): y \in \mathbb{R}^{N}\right\}=m,
$$

where, for a closed bounded set $G \subset \mathbb{R}^{N}$, the capacity of $G$ is defined by

$$
\operatorname{cap} G=\inf \left\{\|u\|_{H^{1}}: u \in H^{1}\left(\mathbb{R}^{N}\right), u \geqslant 1 \text { on } G \text { in the } H^{1} \text {-sense }\right\} .
$$

Moreover, it is easily seen that

$$
\lim _{|a|_{L^{N / 2}} \rightarrow 0} \sup \left\{E_{a}\left(v_{p}(\cdot-y)\right): y \in \mathbb{R}^{N}\right\}=m .
$$

By the asymptotic behaviour stated in (5.5) and (5.6), the following result can be proved by working as in Theorem 4.1 .

Theorem 5.1. Let $h, k \in \mathbb{N}, h+k \neq 0$, and assume that $\Omega$ is an open set in $\mathbb{R}^{N}$ that satisfies Assumption (C). If

$$
\mathcal{D}=\Omega \backslash \bigcup_{i=1}^{h}\left(\bar{\omega}_{i}+\bar{x}_{i}\right), \quad \bar{x}_{i} \in \mathbb{R}^{N},
$$


with $\omega_{i}$ bounded open sets in $\mathbb{R}^{N}$, and a has the form

$$
a(x)=\sum_{j=1}^{k} a_{j}\left(x-\bar{x}_{h+j}\right), \quad x_{h+j} \in \mathbb{R}^{N},
$$

with $a_{j}$ non-negative functions in $L^{N / 2}\left(\mathbb{R}^{N}\right)$ that satisfy assumption (1.2), then there exist

$$
\begin{aligned}
C_{2} & =C_{2}\left(\omega_{1}\right), \\
C_{3} & =C_{3}\left(\omega_{2}\right), \\
& \vdots \\
C_{h} & =C_{h}\left(\omega_{h-1}\right), \\
L_{1} & =L_{1}\left(\omega_{h}\right), \\
L_{2} & =L_{2}\left(a_{1}\right), \\
& \vdots \\
L_{k} & =L_{k}\left(a_{k-1}\right), \\
\bar{x}_{1} & =\bar{x}_{1}\left(\Omega, \omega_{1}\right), \\
& \vdots \\
\bar{x}_{h} & =\bar{x}_{h}\left(\Omega, \omega_{1}, \bar{x}_{1}, \ldots, \omega_{h-1}, \bar{x}_{h-1}, \omega_{h}\right), \\
\bar{x}_{h+1} & =\bar{x}_{h+1}\left(\mathcal{D}, a_{1}\right), \\
& \vdots \\
\bar{x}_{h+k} & =\bar{x}_{h+k}\left(\mathcal{D}, a_{1}, \bar{x}_{h+1}, \ldots, a_{k-1}, \bar{x}_{h+k-1}, a_{k}\right)
\end{aligned}
$$

such that problem $P(a, \mathcal{D})$ has at least $2(k+h)$ distinct solutions $u_{\omega_{1}}, \ldots, u_{\omega_{h}}$, $u_{a_{1}}, \ldots, u_{a_{k}}, u_{i, i+1}, i=1, \ldots, h+k$, whenever $\operatorname{cap} \bar{\omega}_{i}<C_{i}, i=1, \ldots, h$, and $\left|a_{i}\right|_{L^{N / 2}}<L_{i}$, $i=i, \ldots, k$.

In the case of Theorem 5.1, as a consequence of (5.5) and (5.6), the following estimates can be obtained, besides (5.3) and (5.4):

$$
\begin{array}{r}
\lim _{\operatorname{cap} \bar{\omega}_{i} \rightarrow 0} E_{a}\left(\frac{u_{\omega_{i}}}{\left|u_{\omega_{i}}\right|_{L^{p}}}\right)=m, \quad i=1, \ldots, h, \\
\lim _{\left|a_{i}\right|_{L^{N / 2} \rightarrow 0}} E_{a}\left(\frac{u_{a_{i}}}{\left|u_{a_{i}}\right|_{L^{p}}}\right)=m, \quad i=1, \ldots, k .
\end{array}
$$

Observe also that results similar to those stated in Theorems 4.1 and 5.1 can be proved when there are holes that expand and holes that shrink and bumps that increase and bumps that vanish simultaneously. 
Remark 5.2. If in Theorems 4.1 and 5.1 we consider $\Omega=\mathbb{R}^{N}$, then we find that problem $P(a, \mathcal{D})$ has at least $2(h+k)-1$ solutions.

In fact, if we consider Theorem 4.1, for example, we can repeat the proof developed in $\S 4$, with the exception of Step 7, so the desired number of solutions can be found.

Acknowledgements. J.M. is partly supported by FONDECYT no. 1010223 and FONDAP in Applied Mathematics (Chile).

R.M. is supported by the Italian National Research Project 'Metodi variazionali e topologici nello studio di fenomeni non lineari'.

\section{References}

1. R. A. Adams and J. J. F. Fournier, Sobolev spaces, 2nd edn, Pure and Applied Mathematics, Volume 140 (Academic, 2003).

2. A. BAHRi AND P. L. Lions, On the existence of a positive solution of semilinear elliptic equations in unbounded domains, Annls Inst. H. Poincaré Analyse Non Linéaire 14 (1997), 365-413.

3. V. Benci and G. Cerami, Positive solutions of some nonlinear elliptic problems in exterior domains, Arch. Ration. Mech. Analysis 99 (1987), 283-300.

4. V. Benci AND G. Cerami, The effect of the domain topology on the number of positive solutions of nonlinear elliptic problems, Arch. Ration. Mech. Analysis 114 (1991), 79-93.

5. V. Benci, G. Cerami and D. Passaseo, On the number of the positive solutions of some nonlinear elliptic problems, in Nonlinear analysis, Quaderni Scuola Normale Superiore Pisa, pp. 93-107 (Scuola Normale Superiore Pisa, 1991).

6. H. BeRESTYCKi AND P. L. Lions, Nonlinear scalar fields equations, I, Existence of a ground-state, Arch. Ration. Mech. Analysis 82 (1983), 313-346.

7. G. Cerami and R. Molle, Multiple positive solutions for singularly perturbed elliptic problems in exterior domains, Annls Inst. H. Poincaré Analyse Non Linéaire 20 (2003), $759-777$.

8. G. Cerami and R. Molle, A multiplicity result for singularly perturbed problems in nontrivial domains, Adv. Nonlin. Studies 4 (2004), 431-452.

9. G. Cerami And D. PASSASEO, Existence and multiplicity of positive solutions for nonlinear elliptic problems in exterior domains with 'rich' topology, Nonlin. Analysis 18 (1992), 109-119.

10. G. Cerami and D. Passaseo, Existence and multiplicity results for semilinear elliptic Dirichlet problems in exterior domains, Nonlin. Analysis 24 (1995), 1533-1547.

11. G. Cerami, R. Molle and D. Passaseo, Positive solutions of semilinear elliptic problems in unbounded domains with unbounded boundary, Annls Inst. H. Poincaré Analyse Non Linéaire, in press.

12. M. J. Esteban, Nonlinear elliptic problems in strip-like domains: symmetry of positive vortex rings, Nonlin. Analysis 7 (1983), 365-379.

13. M. J. Esteban and P. L. Lions, Existence and nonexistence results for semilinear elliptic problems in unbounded domains, Proc. R. Soc. Edinb. A 93 (1982), 1-14.

14. B. Gidas, W. M. Ni And L. Nirenberg, Symmetry of positive solutions of nonlinear elliptic equations in $\mathbb{R}^{N}$, Mathematical Analysis and Applications, Part A, Advances in Mathematics Supplementary Studies, Volume 7, pp. 369-402 (Academic, 1981).

15. M. Grossi and A. Pistoia, On the effect of critical points of distance function in superlinear elliptic problems, Adv. Diff. Eqns 5 (2000), 1397-1420.

16. M. K. Kwong, Uniqueness of positive solutions of $\Delta u-u+u^{p}=0$, Arch. Ration. Mech. Analysis 105 (1989), 243-266. 
17. R. Molle, Semilinear elliptic problems in unbounded domains with unbounded boundary, Asymp. Analysis 38 (2004), 293-307.

18. R. Molle, Positive solutions for a nonlinear elliptic problem with strong lack of compactness, J. Lond. Math. Soc., in press.

19. R. Molle AND D. PASsaseo, On the behaviour of the solutions for a class of nonlinear elliptic problems in exterior domains, Discrete Contin. Dynam. Syst. 4 (1998), 445-454.

20. R. Molle AND D. PAssaseo, Multiple solutions of nonlinear elliptic Dirichlet problems in exterior domains, Nonlin. Analysis 39 (2000), 447-462.

21. R. Molle, M. Musso and D. Passaseo, Positive solutions for a class of nonlinear elliptic problems in $\mathbb{R}^{N}$, Proc. R. Soc. Edinb. A 130 (2000), 141-166.

22. W. A. Strauss, Existence of solitary waves in higher dimensions, Commun. Math. Phys. 55 (1977), 149-162.

23. M. STRUWE, Variational methods: applications to nonlinear partial differential equations and Hamiltonian systems (Springer, 1990).

24. H.-C. WANG, Palais-Smale approaches to semilinear elliptic equations in unbounded domains, Electron. J. Diff. Eqns (2004), Monograph 06.

25. J. WEI, On the construction of single-peaked solutions to a singularly perturbed semilinear Dirichlet problem, J. Diff. Eqns 129 (1996), 315-333. 